\title{
Counting Bounded Tree Depth Homomorphisms
}

\author{
Martin Grohe \\ RWTH Aachen University
}

\begin{abstract}
We prove that graphs $G, G^{\prime}$ satisfy the same sentences of first-order logic with counting of quantifier rank at most $k$ if and only if they are homomorphismindistinguishable over the class of all graphs of tree depth at most $k$. Here $G, G^{\prime}$ are homomorphism-indistinguishable over a class $\mathcal{F}$ of graphs if for each graph $F \in \mathcal{F}$, the number of homomorphisms from $F$ to $G$ equals the number of homomorphisms from $F$ to $G^{\prime}$.
\end{abstract}

\section{Introduction}

Structural information is captured very well by homomorphism counts. Indeed, an old theorem due to Lovász [30] states that two graphs $G, G^{\prime}$ are isomorphic if and only if $\operatorname{hom}(F, G)=\operatorname{hom}\left(F, G^{\prime}\right)$ for all graphs $F$. Here hom $(F, G)$ denotes the number of homomorphisms from graph $F$ to graph $G$; homomorphisms are mappings between vertices that preserve adjacency. This simple theorem is quite useful and can be seen as a the starting point for the theory of graph limits [9, 31, 32]: by associating each graph $G$ with the vector $\operatorname{HOM}(G):=(\operatorname{hom}(F, G) \mid F$ graph $)$, we map graphs into an infinite dimensional real vector space, which can be turned into a Hilbert space by defining a suitable inner product. This transformation enables us to analyse graphs with methods of linear algebra and functional analysis and, for example, to consider convergent sequences of graphs and their limits, called graphons (see [31]). Vector embeddings of graphs are also crucial for applying machine learning methods to graphs. Notably, there is a close connection between homomorphism counts and so-called graph kernels (e.g. [40, 24]) and graph neural networks (e.g. [33, 36]).

However, not only the full homomorphism vector $\operatorname{HOM}(G)$ of a graph $G$, but also its projections on natural subspaces capture very interesting information about $G$. For a class $\mathcal{F}$ of graphs, we consider the projection

$$
\operatorname{HOM}_{\mathcal{F}}(G):=(\operatorname{hom}(F, G) \mid F \in \mathcal{F})
$$

of $\operatorname{HOM}(G)$ onto the subspace indexed by the graphs in $\mathcal{F}$. Following [8], we call graphs $G, G^{\prime}$ homomorphism-indistinguishable over $\mathcal{F}$ if $\operatorname{HOM}_{\mathcal{F}}(G)=\operatorname{HOM}_{\mathcal{F}}\left(G^{\prime}\right)$. Dvorák [14] 
proved that two graphs are homomorphism-indistinguishable over the class $\mathcal{T}_{k}$ of graphs of tree width at most $k$ if and only if they are not distinguishable by the $k$-dimensional Weisfeiler-Leman algorithm, a well-known combinatorial isomorphism test. As we can always restrict homomorphism vectors to connected graphs without loss of information, this implies that two graphs are homomorphism-indistinguishable over the class $\mathcal{T}$ of trees if and only if they are not distinguishable by the 1-dimensional Weisfeiler-Leman algorithm, which is also known as colour refinement and naive vertex classification. Via well-known characterisations of Weisfeiler-Leman indistinguishability in terms of the solvability of certain natural systems of linear inequalities [2, 20, 34] or systems of polynomial equations or inequalities [3, 6, 19, this also yields algebraic characterisations of homomorphism indistinguishability over classes of bounded tree width. A related algebraic characterisation was obtained for homomorphism indistinguishability over the class of paths [13. It is well-known (though usually phrased differently) that two graphs are homomorphism-indistinguishable over the class of cycles if and only if they are cospectral, that is, their adjacency matrices have the same eigenvalues with the same multiplicities. Böker [7] proved that two graphs are homomorphism-indistinguishable over the class of bipartite graphs if and only if they have isomorphic bipartite double covers. The most recent addition to this picture is a result due to Mančinska and Roberson [35] stating that two graphs are homomorphism-indistinguishable over the class of all planar graphs if and only if they are quantum isomorphic. Quantum isomorphism, introduced in [1, is a complicated notion that is based on similar systems of equations as those characterising homomorphism indistinguishability over graphs of bounded tree width, but with non-commutative variables ranging over the elements of some $C^{*}$-algebra.

What we see emerging is a rich theory connecting combinatorics, structural graph theory, and algebraic graph theory. It turns out that logic is also an integral part of this theory, not only because some of the algebraic characterisations of homomorphism indistinguishability can be phrased in terms of propositional proof complexity [3, 6, [19, but also because there is a well-known characterisation of the Weisfeiler-Leman algorithm and hence homomorphism indistinguishability over classes of bounded tree width in terms of logical equivalence. The logic $C$ is the extension of first-order logic by counting quantifiers of the form $\exists^{\geq p} x$ ("there exists at least $p$ elements $x$ "). Every C-formula is equivalent to a formula of plain first-order logic. However, we are mainly interested in fragments of the logic obtained by restricting the quantifier rank or the number of variables of formulas, and the translation from $C$ to first-order logic preserves neither the quantifier rank nor the number of variables (see Remark 2.11). The logic C and its finite variable fragments have first been considered by Immerman in the 1980s [22, 23, and they have played an important role in finite model theory since then. Cai, Fürer, and Immerman [11] showed that equivalence in the $(k+1)$-variable fragment $\mathrm{C}^{k+1}$ of $\mathrm{C}$ corresponds to indistinguishability by the $k$-dimensional Weisfeiler-Leman algorithm. Thus, two graphs are $C^{k+1}$-equivalent if and only if they are homomorphism indistinguishable over the class $\mathcal{T}_{k}$ of graphs of tree width at most $k$.

Rather than restricting the number of variables in a formula, it is, arguably, even more fundamental to restrict the quantifier rank (maximum number of nested quantifiers in a formula). Our main result is the following characterisation of equivalence in the fragment 
$\mathrm{C}_{k}$ of $\mathrm{C}$ consisting of all formulas of quantifier rank at most $k$.

Theorem 1.1. For all $k \geq 1$ and all graphs $G, G^{\prime}$ the following are equivalent.

(i) $G$ and $G^{\prime}$ are homomorphism-indistinguishable over the class $\mathcal{T} \mathcal{D}_{k}$ of all graphs of tree depth at most $k$.

(ii) $G$ and $G^{\prime}$ satisfy the same $\mathrm{C}_{k}$-sentences.

Tree depth, introduced by Nešetřil and Ossona de Mendez [37], is a structural graph parameter that has received a lot of attention in recent years (e.g. [5, 10, 12, 17, 16]). Our result adds a characterisation of homomorphism indistinguishability over classes of bounded tree depth to the theory of homomorphism indistinguishability sketched above.

However, our result is also interesting from a purely logical point of view. It can be seen simultaneously as a locality theorem and as a quantifier elimination theorem. Locality, because as noted above, when considering homomorphism indistinguishability, we can restrict our attention to connected graphs. Connected graphs of tree depth at most $k$ are known to have a radius of at most $2^{k-1}-1$ (see [38]), and hence their homomorphic images will always be contained in neighbourhoods of radius at most $2^{k-1}-1$. This means that homomorphism indistinguishability over graphs of tree depth $k$ and thus $\mathrm{C}_{k^{-}}$ equivalence only depend on neighbourhoods of radius at most $2^{k-1}-1$. This consequence of our main theorem was known before [27], but we believe that our approach sheds some new light on locality. It should be seen in the context of other recent and not-so-recent locality results for counting logics $[27,28,29,25,26,39$. Let us remark (as already noted by Libkin [27]) that the exact choice of a counting extension of first-order logic is not so important when we only study equivalence between structures 1

Our theorem is a quantifier-elimination result, because it says that we can replace the $k$ nested quantifiers of a $\mathrm{C}_{k}$-formula, which may involve alternations between existential and universal quantifiers, by flat, unnested homomorphism counts. While new in this context, replacing quantifier alternation by counting is a common theme in complexity theory, most prominently represented by Toda's theorem [41] that $\mathrm{P} \# \mathrm{P}$ contains the polynomial hierarchy.

The proof of our theorem is harder than one might expect in view of the numerous previous results on homomorphism indistinguishability. The overall structure of the proof is as follows: in the first step we use linear algebraic techniques that go back to Lovász [30] to show that homomorphism counts can be expressed by counts of more restrictive structure preserving mappings. In the second step, the connection to logic is established via an Ehrenfeucht-Fraïssé game and interpolation techniques. To carry out the first step, we need to prove the invertibility of certain homomorphism matrices, which we achieve by a decomposition into lower-triangular and upper triangular matrices of full rank. The precise nature of this decomposition is what makes the proof difficult; we need to go through various intermediate mappings obeying certain carefully chosen constraints.

${ }^{1}$ The reason is that over a fixed finite graph, formulas of other counting extensions of first-order logic, such as the $\operatorname{logic} \mathrm{FOCN}(\mathbb{P})$ of $[25$, are equivalent to C-formulas of the same quantifier rank. 
The structure of the paper is simple: we prove the theorem and then discuss some of its consequences.

\section{Preliminaries}

\subsection{Graphs and Homomorphisms}

We always assume graphs to be undirected and vertex-coloured. Thus a graph is a triple $\left(V(G), E(G), \gamma^{G}\right)$ where $V(G)$ is a finite set, $E(G) \subseteq\left(\begin{array}{c}V(G) \\ 2\end{array}\right)$, and $\gamma^{G}: V(G) \rightarrow \Gamma$ for some set $\Gamma$ whose elements we view as "colours" 2 The order of a graph is $|G|:=|V(G)|$. A graph $G$ is a subgraph of a graph $H$ (we write $G \subseteq H$ ) if $V(G) \subseteq V(H), E(G) \subseteq E(H)$, and $\gamma^{G}(v)=\gamma^{H}(v)$ for all $v \in V(G)$.

A homomorphism from a graph $F$ to a graph $G$ is a mapping $h: V(F) \rightarrow V(G)$ such that $h(u) h(v) \in E(G)$ for all $u v \in E(F)$ and $\gamma^{F}(u)=\gamma^{G}(h(u))$ for all $u \in V(F)$. We write $h: F \rightarrow G$ to denote that $h$ is a homomorphism from $F$ to $G$. We denote the number of homomorphism from $F$ to $G$ by hom $(F, G)$. Graphs $G, G^{\prime}$ are homomorphismindistinguishable over a class $\mathcal{F}$ of graphs if $\operatorname{hom}(F, G)=\operatorname{hom}\left(F, G^{\prime}\right)$ for all $F \in \mathcal{F}$; otherwise they are homomorphism-distinguishable over $\mathcal{F}$.

Observe that for a disconnected graph $F$ with connected components $F_{1}, \ldots, F_{\ell}$ and for an arbitrary graph $G$ it holds that $\operatorname{hom}(F, G)=\prod_{i=1}^{\ell} \operatorname{hom}\left(F_{i}, G\right)$. This means that if $\mathcal{F}$ is a class of graphs such that all connected components of graphs in $\mathcal{F}$ belong to $\mathcal{F}$ as well, then graphs $G, G^{\prime}$ are homomorphism-indistinguishable over $\mathcal{F}$ if and only if they are homomorphism-indistinguishable over the class $\mathcal{F}^{c}$ of all connected graphs in $\mathcal{F}$.

A homomorphism $h: F \rightarrow G$ is an embedding (or monomorphism) from $F$ to $G$ (we write $h: F \hookrightarrow G$ ) if it is injective. A homomorphism $h: F \rightarrow G$ is an epimorphism from $F$ to $G$ (we write $h: F \rightarrow G$ ) if $h$ is surjective and for every edge $v v^{\prime} \in E(G)$ there is an edge $u u^{\prime} \in E(F)$ such that $h(u)=v$ and $h\left(u^{\prime}\right)=v^{\prime}$. (Note that not every surjective homomorphism is an epimorphism.) If $H: F \rightarrow G$ is an epimorphism, then $G$ is a homomorphic image of $F$. By $\operatorname{emb}(F, G)$ and epi $(F, G)$ we denote the numbers of embeddings and epimorphisms from $F$ to $G$.

If $\pi$ is a partial mapping from $V(F)$ to $V(G)$, then by $\operatorname{hom}(F, G ; \pi)$ we denote the number of homomorphisms from $F$ to $G$ that extend $\pi$. In particular, for vertices $u \in V(F)$ and $v \in V(G)$, by hom $(F, G ; u \mapsto v)$ we denote the number of homomorphism $h: F \rightarrow G$ with $h(u)=v$. We use similar notations for embeddings, epimorphisms, and other types of mappings that we shall introduce later.

\subsection{First-Order Logic with Counting}

To define the syntax of the logic $\mathrm{C}$, we assume that we have an infinite supply of variables, which we denote by $x, y, z$ and variants such as $x^{\prime}, y_{1}$. Variables range over the vertices of a graph. Atomic formulas (in the language of graphs) are of the form $x=y, E(x, y)$ ("there is an edge between $x, y$ "), and $\gamma(x)=c$ for colours $c$ (" $x$ has colour $c$ "). C-

\footnotetext{
${ }^{2}$ For clarity of the presentation, we decided to focus on undirected graphs here. The result can be
} extended to arbitrary relational structures, see Section 5.3 for a brief discussion. 
formulas are constructed from atomic formulas using negation $\neg \varphi$, disjunction $(\varphi \vee \psi)$, and counting quantifiers $\exists^{\geq p} x \varphi$, where $p \in \mathbb{N}, x$ is a variable, and $\varphi, \psi$ are formulas.

An occurrence of a variable $x$ is free in a formula $\varphi$ if it is outside the range of all quantifications $\exists^{\geq p} x$. A sentence is a formula without any free variables. We often write $\varphi\left(x_{1}, \ldots, x_{\ell}\right)$ to indicate that the free variables of $\varphi$ are among $x_{1}, \ldots, x_{\ell}$. (Not all of these variables are required to appear in $\varphi$.) For a formula $\varphi\left(x_{1}, \ldots, x_{\ell}\right)$, a graph $G$, and vertices $v_{1}, \ldots, v_{\ell} \in V(G)$, we write $G \models \varphi\left(v_{1}, \ldots, v_{\ell}\right)$ to denote that $G$ satisfies $\varphi$ if the variables $x_{i}$ are interpreted by the vertices $v_{i}$. We also write $\varphi(\boldsymbol{x})$ and $\varphi(\boldsymbol{v})$ for tuples $\boldsymbol{x}=\left(x_{1}, \ldots, x_{\ell}\right), \boldsymbol{v}=\left(v_{1}, \ldots, v_{\ell}\right)$. Now we can define the semantics of the logic C inductively in the obvious way. In particular, for $\varphi\left(y_{1}, \ldots, y_{\ell}\right)=\exists \geq p x \psi\left(x, y_{1}, \ldots, y_{\ell}\right)$ we let $G \models \varphi\left(w_{1}, \ldots, w_{\ell}\right)$ if there are mutually distinct $v_{1}, \ldots, v_{p} \in V(G)$ such that $G \models \psi\left(v_{i}, w_{1}, \ldots, w_{\ell}\right)$ for all $i \in[p]$.

The quantifier rank $\operatorname{qr}(\varphi)$ of a C-formula $\varphi$ is defined inductively by letting $\operatorname{qr}(\varphi):=0$ for all atomic formulas $\varphi$ and $\operatorname{qr}(\neg \varphi):=\operatorname{qr}(\varphi), \operatorname{qr}(\varphi \vee \psi):=\max \{\operatorname{qr}(\varphi), \operatorname{qr}(\psi)\}$, and $\operatorname{qr}\left(\exists^{\geq p} x \varphi\right):=\operatorname{qr}(\varphi)+1$. By $C_{k}$ we denote the fragment of $\mathrm{C}$ consisting of all formulas of quantifier rank at most $k$. Graphs $G, G^{\prime}$ are $C_{k}$-equivalent if $G \models \varphi \Longleftrightarrow G^{\prime} \models \varphi$ for all $\mathrm{C}_{k}$-sentences $\varphi$. We write $G \equiv_{k}^{C} G^{\prime}$ to denote that $G$ and $G^{\prime}$ are $\mathrm{C}_{k}$-equivalent We extend this notation to formulas with free variables, writing $G, \boldsymbol{v} \equiv_{k}^{\mathrm{C}} G^{\prime}, \boldsymbol{v}^{\prime}$ for tuples $\boldsymbol{v} \in V(G)^{\ell}, \boldsymbol{v}^{\prime} \in V\left(G^{\prime}\right)^{\ell}$ to denote that for all $\mathrm{C}_{k}$-formulas $\varphi(\boldsymbol{x})$ it holds that $G \models \varphi(\boldsymbol{v}) \Longleftrightarrow G^{\prime} \models \varphi\left(\boldsymbol{v}^{\prime}\right)$.

Remark 2.1. Interpreting the usual existential quantifier $\exists$ as $\exists \geq 1$, we can view firstorder logic $\mathrm{FO}$ as a fragment of $\mathrm{C}$. Observe that $\mathrm{C}$ has the same expressive power as its fragment FO, because $\exists^{\geq} x \varphi\left(x, y_{1}, \ldots, y_{\ell}\right)$ can be equivalently expressed as

$$
\exists x_{1} \ldots \exists x_{p}\left(\bigwedge_{1 \leq i<j \leq p} \neg x_{i}=x_{j} \wedge \bigwedge_{1 \leq i \leq p} \varphi\left(x_{i}, y_{1}, \ldots, y_{\ell}\right)\right) .
$$

However, this increases the quantifier rank. It is easy to see that for every $k \geq 1$, $\mathrm{C}_{k}$ is strictly more expressive than the fragment $\mathrm{FO}_{k}$ of first-order logic consisting of all formulas of quantifier rank at most $k$. Actually, for every $k$ the $\mathrm{C}_{1}$-formula $\exists^{\geq k+1} x(x=x)$ is not equivalent to any $\mathrm{FO}_{k}$-formula.

\subsection{The Bijective Pebble Game}

The bijective pebble game, introduced by Hella [21], gives a combinatorial characterisation of equivalence in the logic $C$ and its fragments $C_{k}$.

Let $G, G^{\prime}$ be graphs of the same order. The bijective pebble game on $G$ and $G^{\prime}$ is played by two players called Spoiler and the Duplicator. Positions of the game are pairs $\left(\boldsymbol{v}, \boldsymbol{v}^{\prime}\right)$ where $\boldsymbol{v} \in V(G)^{k}, \boldsymbol{v}^{\prime} \in V\left(G^{\prime}\right)^{k}$ for some $k \geq 0$. A play of the game consists of a sequence of rounds, starting from some initial position $\left(\boldsymbol{v}_{0}, \boldsymbol{v}_{0}^{\prime}\right)$, where $\boldsymbol{v}_{0}=\left(v_{1}, \ldots, v_{\ell}\right)$ and $\boldsymbol{v}_{0}^{\prime}=\left(v_{1}^{\prime}, \ldots, v_{\ell}^{\prime}\right)$ for some $\ell \geq 0$. The default initial position is the "empty position" $((),())$. In round $i$ of the game, Duplicator chooses a bijection $f_{i}: V(G) \rightarrow V\left(G^{\prime}\right)$. Then Spoiler chooses a $v_{\ell+i} \in V(G)$, and we let $v_{\ell+i}^{\prime}:=f_{i}\left(v_{\ell+i}\right)$. The position after round 
$i$ is $\left(\boldsymbol{v}_{i}, \boldsymbol{v}_{i}^{\prime}\right):=\left(\left(v_{1}, \ldots, v_{\ell+i}\right),\left(v_{1}^{\prime}, \ldots, v_{\ell+i}^{\prime}\right)\right)$. In the $k$-round game, the play ends after $k$-rounds, and Duplicator wins the play if $\boldsymbol{v}_{k} \mapsto \boldsymbol{v}_{k}^{\prime}:=\left(v_{i} \mapsto v_{i}^{\prime} \mid 1 \leq i \leq k+\ell\right)$ is a local isomorphism from $G$ to $G^{\prime}$, that is, for all $i, j \in[\ell+k]$ the following conditions are satisfied:

- $v_{i}=v_{j} \Longleftrightarrow v_{i}^{\prime}=v_{j}^{\prime}$;

- $v_{i} v_{j} \in E(G) \Longleftrightarrow v_{i}^{\prime} v_{j}^{\prime} \in E\left(G^{\prime}\right)$;

- $\gamma^{G}\left(v_{i}\right)=\gamma^{G^{\prime}}\left(v_{i}^{\prime}\right)$.

If $\boldsymbol{v}_{k} \mapsto \boldsymbol{v}_{k}^{\prime}$ is not a local isomorphism, then Spoiler wins the play.

We can now define winning strategies for Spoiler and Duplicator in the usual way.

The following lemma, which links the bijective pebble game to the logic C, is a minor variant of a theorem due to Hella 21] and of the standard characterisation of first-order logic in terms of Ehrenfeucht-Fraïssé games (see, for example, [15]).

Lemma 2.2. For all $k, \ell \geq 0$, all graphs $G, G^{\prime}$ of the same order, and all $\boldsymbol{v} \in V(G)^{\ell}, \boldsymbol{v}^{\prime} \in$ $V\left(G^{\prime}\right)^{\ell}$ the following are equivalent.

(i) Duplicator has a winning strategy for the k-round bijective pebble game on $G, G^{\prime}$ with initial position $\left(\boldsymbol{v}, \boldsymbol{v}^{\prime}\right)$.

(ii) $G, \boldsymbol{v} \equiv{ }_{k}^{\mathrm{C}} G^{\prime}, \boldsymbol{v}^{\prime}$.

If we do not specify the initial position of the game, we always assume it is the empty position $((),(())$. Thus the lemma implies that Duplicator has a winning strategy for the $k$-round bijective pebble game on $G, G^{\prime}$ if and only if $G \equiv_{k}^{\mathrm{C}} G^{\prime}$.

\subsection{Graphs of Bounded Tree Depth}

It will be convenient in this paper to view trees and forests as partially ordered sets. A forest $S$ is a pair $\left(V(S), \preceq^{S}\right)$ consisting of a (finite) vertex set $V(S)$ and a partial order $\preceq^{S}$ on $V(S)$ such that for every $t \in V(S)$ the set $\{u \in V(S) \mid u \preceq S t\}$ is a chain, that is, its elements are pairwise comparable. We denote the strict partial order associated with $\preceq^{S}$ by $\prec^{S}$. If $t \prec^{S} u$ and there is no $v \in V(S)$ such that $t \prec^{S} v$ and $v \prec^{S} u$, then we say that $u$ is a child of $t$ and that $t$ is the parent of $u$. This gives us a one-to-one correspondence between forests viewed as partially ordered sets and rooted forests in the usual graph-theoretic sense. The $\preceq^{S}$ minimal elements of $V(S)$ are called the roots of $S$. The height of $S$ is the length $|X|$ of the longest chain $X$ in $S$. Note that, differing from the standard graph theoretic definition, we count the number of vertices (and not the number of edges) on a path from the root to a leaf. In particular, a forest consisting of roots only has height 1 .

A forest $T$ with a unique root is a tree. We denote the root of a tree $T$ by $r^{T}$. A subtree of a tree $T$ is a tree $T^{\prime}$ with $V\left(T^{\prime}\right) \subseteq V(T)$ such that $\preceq^{T^{\prime}}$ is the restriction of $\preceq^{T}$ to $V\left(T^{\prime}\right)$. Thus a subtree is an induced substructure that is a tree itself. Observe that a set $U \subseteq V(T)$ induces a subtree of $T$ if and only if $U$ has a unique $\preceq^{T}$-minimal element. This 
notion of subtree does not coincide with the usual graph-theoretic notion of a subtree of a tree. In particular, elements of a subtree can be interleaved with elements that do not belong to the subtree.

An elimination forest of a graph $G$ is a forest $S$ such that $V(S)=V(G)$ and for every edge $u v \in E(G)$, either $u \preceq^{S} v$ or $v \preceq^{S} u$. If an elimination forest $S$ of $G$ is a tree, we also call it an elimination tree of $G$. The tree depth of a graph $G$ is the minimum $k$ such that $G$ has an elimination forest of height $k$. We denote the class of all graphs of tree depth at most $k$ by $\mathcal{T} \mathcal{D}_{k}$ and the class of all connected graphs in $\mathcal{T} \mathcal{D}_{k}$ by $\mathcal{T} \mathcal{D}_{k}^{c}$.

\section{Lemma 2.3 (Nešetřil and Ossona de Mendez [37]).}

(1) $\mathcal{T} \mathcal{D}_{1}^{c}$ consists of all 1-vertex graphs.

(2) For $k \geq 1, \mathcal{T D}_{k+1}^{c}$ is the class of all connected graphs $F$ that have a vertex $r$ such that all connected components of $F \backslash\{r\}$ are in $\mathcal{T D}_{k}^{c}$.

(3) For all $k \geq 1, \mathcal{T D}_{k}$ is the class of disjoint unions of graphs in $\mathcal{T D}_{k}^{c}$.

We let $\mathcal{D}$ be the class of all pairs $(F, T)$ where $F$ is a graph and $T$ an elimination tree of $F$. We usually denote elements of $\mathcal{D}$ by $D$

For $D=(F, T) \in \mathcal{D}$, we let $F^{D}:=F, T^{D}:=T$ and $V(D):=V(F)=V(T)$, $E(D):=E(F), \gamma^{D}:=\gamma^{F}, \preceq^{D}:=\preceq^{T}$, and $r^{D}:=r^{T}$. We call $r^{D}$ the root of $D$. The height of $D$ is the height of $T^{D}$. We denote the class of all $D \in \mathcal{D}$ of height at most $k$ by $\mathcal{D}_{k}$. Observe that a connected graph $F$ is in $\mathcal{T} \mathcal{D}_{k}$ if and only if there is a $D \in \mathcal{D}_{k}$ such that $F^{D}=F$.

Remark 2.4. There is a strange asymmetry in the definition of $\mathcal{D}$ : for pairs $(F, T) \in \mathcal{D}$, we require $T$ to be a tree, not an arbitrary forest, but we do not require the graph $F$ to be connected. Yet this definition is carefully chosen. In particular, if we required $F$ to be connected then we would run into difficulties in the proof of Lemma 4.6.

\section{Past-Preserving Homomorphisms}

Let $D \in \mathcal{D}_{k}$, and let $G$ be an arbitrary graph. A homomorphism from $D$ to $G$ is simply a homomorphism from $F^{D}$ to $G$. We write $h: D \rightarrow G$ to denote that $h$ is a homomorphism from $D$ to $G$, and we let $\operatorname{hom}(D, G):=\operatorname{hom}\left(F^{D}, G\right)$ be the number of homomorphisms from $D$ to $G$. A homomorphism $h: D \rightarrow G$ is an epimorphism (we write $h: D \rightarrow G$ ) if it is an epimorphism from $F^{D}$ to $G$.

A homomorphism $h: D \rightarrow G$ is past-injective if for all $u, v \in V(D)$ with $u \prec^{D} v$ we have $h(u) \neq h(v)$. If in addition, for all $u, v \in V(D)$ with $u \preceq^{D} v$ we have $u v \in$ $E(D) \Longleftrightarrow h(u) h(v) \in E(G)$, then $h$ is past-preserving. We denote the number of past-injective homomorphisms from $D$ to $G$ by pi-hom $(D, G)$ and the number of pastpreserving homomorphisms from $D$ to $G$ by $\operatorname{pp-hom}(D, G)$. In this section, we shall prove that we can compute the numbers of past-preserving homomorphisms to a graph

${ }^{3}$ The reader may wonder why we chose the letter "d" (in $D$ and $\mathcal{D}$ ). One reason is that it picks up the "d" in depth and that $\mathcal{D}$ is close to $\mathcal{T} \mathcal{D}$. Or think of "d" as standing for "decomposed graph". 
from the numbers of homomorphisms and vice versa. The difficult first step will be to establish an equivalence between the numbers of past-injective homomorphisms and homomorphisms.

The general strategy for establishing such an equivalence, going back to Lovász [30], is to establish a linear relationship between the corresponding counting vectors, in our case the vectors $\operatorname{HOM}_{\mathcal{T D}_{k}}(G)=\left(\operatorname{hom}(F, G) \mid F \in \mathcal{T D}_{k}\right)$ and the corresponding vector of past-injective homomorphism counts and then show that the matrix relating the two vectors is invertible (this will happen in Lemma 3.2, Corollary 3.3, and Lemma 3.5). On the linear algebra side, we shall write the (infinite) matrix of homomorphism counts as a product of an upper-triangular matrix with nonzero diagonal entries and a lowertriangular matrix with nonzero diagonal entries. This decomposition of the homomorphism matrix corresponds to a decomposition of homomorphisms. The upper triangular matrix is obtained by considering some form of injective homomorphisms, in our case past-injective homomorphisms. The lower triangular matrix corresponds to suitable surjective homomorphisms, in our case shrinking epimorphisms, to be introduced next. The reason that we cannot just work with plain injective and surjective homomorphisms (or rather epimorphisms) is that the homomorphic image of a graph of tree depth at most $k$ may have larger tree depth than $k$. However, we shall prove (in Lemma 3.1) that shrinking epimorphisms preserve tree depth.

Let $D \in \mathcal{D}_{k}$, and let $G$ be a graph with $V(G) \subseteq V(D)$ (but not necessarily $G \subseteq F^{D}$ ). A shrinking homomorphism from $D$ to $G$ is a homomorphism $h: D \rightarrow G$ such that $h(u) \preceq^{D} u$ for all $u \in V(D)$ and $h$ is idempotent, that is, $h(h(u))=h(u)$ for all $u \in V(D)$. We are mainly interested in shrinking epimorphisms. We denote the number of shrinking epimorphism from $D$ to $G$ by s-epi $(D, G)$. Note that if $h$ is a shrinking epimorphism from $D$ to $G$ then $h(v)=v$ for all $v \in V(G)$. Indeed, since $h$ is surjective, we have $v=h(u)$ for some $u$ and therefore $h(v)=h(h(u))=h(u)=v$. This implies that for all $v \in V(G)$ we have $\gamma^{G}(v)=\gamma^{D}(v)$.

To simplify the notation, for graphs $F, G$ we write $G \sqsubseteq_{\gamma} F$ if $V(G) \subseteq V(F)$ and $\gamma^{G}(v)=\gamma^{F}(v)$ for all $v \in V(G)$. For a $D \in \mathcal{D}$ we write $G \sqsubseteq_{\gamma} D$ instead of $G \sqsubseteq_{\gamma} F^{D}$.

Lemma 3.1. Let $D \in \mathcal{D}_{k}, G \sqsubseteq_{\gamma} D$, and let $f: D \rightarrow G$ be a shrinking epimorphism from $D$ to $G$. Then $T^{D}$ induces a subtree on $V(G)$, and this subtree $T^{D}[V(G)]$ is an elimination tree of $G$ of height at most $k$, that is, $\left(G, T^{D}[V(G)]\right) \in \mathcal{D}_{k}$.

Proof. We first prove that $T^{\prime}:=T^{D}[V(G)]$ is a tree of height at most $k$. Observe that $f\left(r^{D}\right)=r^{D}$ and thus $r^{D} \in V(G)$. Hence $V(G)$ has a unique $\preceq^{D}$-minimal element, and $T^{\prime}$ is a tree. Clearly, the height of $T^{\prime}$ is at most the height of $T^{D}$ and hence at most $k$.

It remains to prove that $T^{\prime}$ is an elimination tree of $G$. Let $v v^{\prime} \in E(G)$. We shall prove that either $v \preceq^{T^{\prime}} v^{\prime}$ or $v^{\prime} \preceq^{T^{\prime}} v$. Since $f$ is an epimorphism, there is an edge $u u^{\prime} \in E(D)$ such that $f(u)=v$ and $f\left(u^{\prime}\right)=v^{\prime}$. Then $v \preceq^{D} u$ and $v^{\prime} \preceq^{D} u^{\prime}$. Since $T^{D}$ is an elimination tree of $F^{D}$, either $u \preceq^{D} u^{\prime}$ or $u^{\prime} \preceq^{D} u$. Without loss of generality we assume $u \preceq^{D} u^{\prime}$. Then $v, v^{\prime} \preceq^{D} u^{\prime}$. Since the set $\left\{t \in V(D) \mid t \preceq^{D} u^{\prime}\right\}$ is a chain in the tree $T^{\bar{D}}$, either $v \preceq^{D} v^{\prime}$ or $v^{\prime} \preceq^{D} v$. As $\preceq^{T^{\prime}}$ is the restriction of $\preceq^{D}$ to $V(G)$, this implies that $v \preceq^{T^{\prime}} v^{\prime}$ or $v^{\prime} \preceq^{T^{\prime}} v$. 
Lemma 3.2. Let $D \in \mathcal{D}_{k}$, and let $h: D \rightarrow H$ be a homomorphism from $D$ to some graph $H$. Then there is a graph $G \sqsubseteq_{\gamma} D$, a shrinking epimorphism $f: D \rightarrow G$, and a past-injective homomorphism $g:\left(G, T^{D}[V(G)]\right) \rightarrow H$ such that $h=g \circ f$.

Furthermore, $G, f$, and $g$ are unique. That is, if $G^{\prime} \sqsubseteq_{\gamma} D$ and $f^{\prime}: D \rightarrow G^{\prime}$ is a shrinking epimorphism and $g:\left(G^{\prime}, T^{D}\left[V\left(G^{\prime}\right)\right]\right) \rightarrow H$ is past-injective such that $h=$ $g^{\prime} \circ f^{\prime}$, then $G=G^{\prime}, f=f^{\prime}$, and $g=g^{\prime}$.

Proof. Let $W:=h(V(D)) \subseteq V(H)$ be the range of $h$. Then the sets $h^{-1}(w)$, for $w \in W$, form a partition of $V(D)$. For every $u \in V(D)$, let $f(u)$ be the $\preceq^{D}$-minimal element in $h^{-1}(h(u)) \cap\left\{t \mid t \preceq^{D} u\right\}$. There is at most one such element because $\left\{t \mid t \preceq^{D} u\right\}$ is a chain. Note that $f$ is idempotent. Let $G:=f\left(F^{D}\right)$ be the graph with vertex set $V(G):=f(V(D))$ and edge set $E(G):=\left\{f(u) f\left(u^{\prime}\right) \mid u u^{\prime} \in E(D)\right\}$. Then $f: D \rightarrow G$ is a shrinking epimorphism. Hence by Lemma 3.1, the induced subtree $T^{D}[V(G)]$ is an elimination tree of $G$ of height at most $k$.

For all $u, u^{\prime} \in V(D)$, if $f(u)=f\left(u^{\prime}\right)$ then $h(u)=h\left(u^{\prime}\right)$. Thus there is a mapping $g: V(G) \rightarrow V(H)$ such that $h=g \circ f$. As $h$ is a homomorphism and $G=f(F)$, the mapping $g$ is a homomorphism from $G$ to $H$. Indeed, for every edge $v v^{\prime} \in E(G)$ there is an edge $u u^{\prime} \in E(D)$ such that $f(u)=v$ and $f\left(u^{\prime}\right)=v^{\prime}$. Then $g(v) g\left(v^{\prime}\right)=h(u) h\left(u^{\prime}\right) \in$ $E(H)$.

To prove that $g$ is past-injective, suppose for contradiction that there are $v, v^{\prime} \in V(G)$ such that $v \prec^{T^{D}}[V(G)] v^{\prime}$ and $g(v)=g\left(v^{\prime}\right)=: w$. Note that $v \prec^{T^{D}[V(G)]} v^{\prime}$ implies $v \prec^{D} v^{\prime}$. As $f$ is the identity on $V(G) \subseteq V\left(F^{D}\right)$, we have $h(v)=h\left(v^{\prime}\right)=w$. By the definition of $f$, this means that $v=f(v)$ and $v^{\prime}=f\left(v^{\prime}\right)$ are $\preceq^{D}$-minimal elements in $h^{-1}(w)$. Since $v \neq v^{\prime}$, it follows that $v \swarrow^{D} v^{\prime}$. This is a contradiction.

It remains to prove the uniqueness. Let $G^{\prime} \sqsubseteq_{\gamma} D$ and $f^{\prime}: D \rightarrow G$ a shrinking epimorphism and $g^{\prime}:\left(G^{\prime}, T\left[V\left(G^{\prime}\right)\right]\right) \rightarrow H$ a past-injective homomorphism such that $h=g^{\prime} \circ f^{\prime}$. If $f=f^{\prime}$ then $G=G^{\prime}$, because $G^{\prime}=f\left(F^{D}\right)=f^{\prime}\left(F^{D}\right)=G$, and $g=g^{\prime}$ because $g \circ f=g^{\prime} \circ f^{\prime}$ and $f, f^{\prime}$ are surjective. Suppose for contradiction that $f^{\prime} \neq f$. Let $u \in V(D)$ such that $f^{\prime}(u) \neq f(u)$ and, subject to this condition, $u$ is $\preceq^{D}$-minimal.

Case 1: $f(u) \neq u$.

Let $u^{\prime}:=f(u)$. Then $u^{\prime} \prec^{D} u$ and, since $f$ is idempotent, $f\left(u^{\prime}\right)=u^{\prime}$. Thus

$$
h(u)=g(f(u))=g\left(u^{\prime}\right)=g\left(f\left(u^{\prime}\right)\right)=h\left(u^{\prime}\right) .
$$

By the minimality of $u$, we have $f^{\prime}\left(u^{\prime}\right)=f\left(u^{\prime}\right)=u^{\prime}$. This implies

$$
g^{\prime}\left(f^{\prime}(u)\right)=h(u)=h\left(u^{\prime}\right)=g^{\prime}\left(f^{\prime}\left(u^{\prime}\right)\right) .
$$

Since $f^{\prime}(u) \preceq^{D} u$ and $f^{\prime}\left(u^{\prime}\right)=u^{\prime} \preceq^{D} u$ and $T^{D}$ is a tree, either $f^{\prime}(u) \preceq^{D} f^{\prime}\left(u^{\prime}\right)$ or $f^{\prime}\left(u^{\prime}\right) \preceq^{D} f^{\prime}(u)$. Since $g^{\prime}$ is past-injective, by (3.A) we have neither $f^{\prime}(u) \prec^{D} f^{\prime}\left(u^{\prime}\right)$ nor $f^{\prime}\left(u^{\prime}\right) \prec^{D} f^{\prime}(u)$ and thus $f^{\prime}(u)=f^{\prime}\left(u^{\prime}\right)=u^{\prime}=f(u)$. This is a contradiction.

Case 2: $f(u)=u$.

Let $u^{\prime}:=f^{\prime}(u)$. Then $u^{\prime} \prec^{D} u$. Since $f^{\prime}$ is idempotent, we have $f^{\prime}\left(u^{\prime}\right)=$ $f^{\prime}\left(f^{\prime}(u)\right)=f^{\prime}(u)=u^{\prime}$ and thus $h(u)=g^{\prime}\left(f^{\prime}(u)\right)=g^{\prime}\left(u^{\prime}\right)=g^{\prime}\left(f^{\prime}\left(u^{\prime}\right)\right)=h\left(u^{\prime}\right)$. By 
the minimality of $u$ we have $f\left(u^{\prime}\right)=f^{\prime}\left(u^{\prime}\right)=u^{\prime}$. Hence $g(u)=g(f(u))=h(u)=$ $h\left(u^{\prime}\right)=g\left(f\left(u^{\prime}\right)\right)=g\left(u^{\prime}\right)$, which contradicts $g$ being past-injective.

Corollary 3.3. Let $D \in \mathcal{D}_{k}$, and let $H$ be a graph. Then

$$
\operatorname{hom}(D, H)=\sum_{G \sqsubseteq \gamma D} \operatorname{s-epi}(D, G) \cdot \operatorname{pi}-\operatorname{hom}\left(\left(G, T^{D}[(V(G)]), H\right) .\right.
$$

Corollary 3.4. Let $D \in \mathcal{D}_{k}$, and let $H, H^{\prime}$ be graphs such that for all $G \sqsubseteq_{\gamma} D$ with $\left(G, T^{D}[V(G)]\right) \in \mathcal{D}_{k}$ it holds that

$$
\text { pi-hom }\left(\left(G, T^{D}[V(G)]\right), H\right)=\operatorname{pi-hom}\left(\left(G, T^{D}[V(G)]\right), H^{\prime}\right) .
$$

Then

$$
\operatorname{hom}(D, H)=\operatorname{hom}\left(D, H^{\prime}\right) .
$$

Proof. Here we use Lemma 3.1 to see that we can restrict the sum in (3.B) to $G$ with $\left(G, T^{D}[V(G)]\right) \in \mathcal{D}_{k}$.

Lemma 3.5. Let $(G, T) \in \mathcal{D}_{k}$, and let $H, H^{\prime}$ be graphs such that for all $F \in \mathcal{T D}_{k}$ with $F \sqsubseteq_{\gamma} G$,

$$
\operatorname{hom}(F, H)=\operatorname{hom}\left(F, H^{\prime}\right) \text {. }
$$

Then

$$
\text { pi-hom }((G, T), H)=\operatorname{pi-hom}\left((G, T), H^{\prime}\right) .
$$

Proof. Let $\mathcal{G}$ be the set of all $\left(G^{\prime}, T^{\prime}\right) \in \mathcal{D}_{k}$ such that $G^{\prime} \sqsubseteq_{\gamma} G$ and $T^{\prime}=T\left[V\left(G^{\prime}\right)\right]$. In particular, $(G, T) \in \mathcal{G}$.

Let $\left(G_{1}, T_{1}\right), \ldots,\left(G_{m}, T_{m}\right)$ be an enumeration of $\mathcal{G}$ such that $\left(G_{m}, T_{m}\right)=(G, T)$ and $\left|G_{i}\right| \leq\left|G_{j}\right|$ for $i \leq j$. Observe that s-epi $\left(\left(G_{i}, T_{i}\right), G_{i}\right)=1$ for all $i$ and that s-epi $\left(\left(G_{i}, T_{i}\right), G_{j}\right)>0$ for $j \neq i$ only if $V\left(G_{j}\right) \subset V\left(G_{i}\right)$ and hence $j<i$. Let $A \in \mathbb{R}^{m \times m}$ be the matrix with entries $A_{i j}:=$ s-epi $\left(\left(G_{i}, T_{i}\right), G_{j}\right)$. Then $A$ is a lower triangular matrix with diagonal entries $A_{i i}=1$ for all $i$. This implies that $A$ is invertible.

Let $\boldsymbol{c}=\left(c_{1}, \ldots, c_{m}\right)^{T}$ be the vector with entries $c_{i}:=\operatorname{hom}\left(G_{i}, H\right)$, and let $\boldsymbol{b}=$ $\left(b_{1}, \ldots, b_{m}\right)^{T}$ be the vector with entries $b_{i}:=$ pi-hom $\left(\left(G_{i}, T_{i}\right), H\right)$. By Corollary 3.3, for every $i$ we have

$$
\begin{aligned}
c_{i} & =\operatorname{hom}\left(\left(G_{i}, T_{i}\right), H\right) \\
& =\sum_{G^{\prime} \sqsubseteq \gamma G_{i}} \mathrm{~s}-\mathrm{epi}\left(\left(G_{i}, T_{i}\right), G^{\prime}\right) \cdot \operatorname{pi}-\operatorname{hom}\left(\left(G^{\prime}, T_{i}\left[V\left(G^{\prime}\right)\right]\right), H\right) \\
& =\sum_{j=1}^{m} A_{i j} b_{j} .
\end{aligned}
$$

Thus $\boldsymbol{c}=A \boldsymbol{b}$, and since $A$ is invertible, $\boldsymbol{b}=A^{-1} \boldsymbol{c}$.

Now let $\boldsymbol{c}^{\prime}=\left(c_{1}^{\prime}, \ldots, c_{m}^{\prime}\right)^{T}$ be the vector with entries $c_{i}^{\prime}:=\operatorname{hom}\left(G_{i}, H^{\prime}\right)$, and let $\boldsymbol{b}^{\prime}=\left(b_{1}^{\prime}, \ldots, b_{m}^{\prime}\right)^{T}$ be the vector with entries $b_{i}^{\prime}:=\operatorname{pi}$-hom $\left(\left(G_{i}, T_{i}\right), H^{\prime}\right)$. Then $\boldsymbol{b}^{\prime}=A^{-1} \boldsymbol{c}^{\prime}$.

By the assumption of the lemma, we have $\boldsymbol{c}=\boldsymbol{c}^{\prime}$. Thus $\boldsymbol{b}=\boldsymbol{b}^{\prime}$. In particular,

$$
\operatorname{pi-hom}((G, T), H)=b_{m}=b_{m}^{\prime}=\operatorname{pi-hom}\left((G, T), H^{\prime}\right) \text {. }
$$


Let us now move on to past-preserving homomorphisms.

Lemma 3.6. Let $D \in \mathcal{D}_{k}$, and let $h: D \rightarrow H$ be a past-injective homomorphism from $D$ to a graph $H$. Then there is a unique graph $G \supseteq F^{D}$ with $V(G)=V(D)$ such that $T^{D}$ is an elimination tree of $G$ and $h$ is a past-preserving homomorphism from $\left(G, T^{D}\right)$ to $H$.

Proof. Suppose that $D=(F, T)$. We let $G$ be the graph with $V(G):=V(F)$,

$$
E(G):=\left\{u v \in\left(\begin{array}{c}
V(F) \\
2
\end{array}\right) \mid u \preceq^{T} v \text { and } h(u) h(v) \in E(H)\right\},
$$

and $\gamma^{G}:=\gamma^{F}$. Then $G \supseteq F$, because $h$ is a homomorphism, and $T$ is an elimination tree of $G$, because $u v \in E(G)$ implies $u \preceq^{T} v$ or $v \preceq^{T} u$. Moreover, $h$ is past-preserving, because it is past-injective and for $u \preceq^{T} v$ we have $u v \in E(G) \Longleftrightarrow h(u) h(v) \in E(H)$.

It remains to prove the uniqueness. Let $G^{\prime} \supseteq F$ with $V\left(G^{\prime}\right)=V(F)$ such that $T$ is an elimination tree of $G^{\prime}$ and $h$ is a past-preserving homomorphism from $\left(G^{\prime}, T\right)$ to $H$. Then $\gamma^{G^{\prime}}=\gamma^{F}=\gamma^{G}$, because $h$ is a homomorphism from $G^{\prime}$ to $H$. Moreover, for all $u v \in E\left(G^{\prime}\right)$, either $u \preceq^{T} v$ or $v \preceq^{T} u$, because $T$ is an elimination tree of $G^{\prime}$, and $u v \in E\left(G^{\prime}\right) \Longleftrightarrow h(u) h(v) \in E(H)$, because $h$ is past-preserving. Thus $E\left(G^{\prime}\right)=E(G)$ and therefore $G=G^{\prime}$.

Corollary 3.7. Let $D \in \mathcal{D}_{k}$, and let $H$ be a graph. Then

$$
\operatorname{pi-hom}(D, H)=\sum_{\substack{G \supseteq F^{D} \\ \text { such that } V(G)=V(D) \\ \text { and }\left(G, T^{D}\right) \in \mathcal{D}_{k}}} \operatorname{pp-hom}\left(\left(G, T^{D}\right), H\right) .
$$

Corollary 3.8. Let $D \in \mathcal{D}_{k}$, and let $H, H^{\prime}$ be graphs such that for all $G \supseteq F^{D}$ with $V(G)=V(D)$ and $\left(G, T^{D}\right) \in \mathcal{D}_{k}$,

$$
\operatorname{pp}-\operatorname{hom}\left(\left(G, T^{D}\right), H\right)=\operatorname{pp}-\operatorname{hom}\left(\left(G, T^{D}\right), H^{\prime}\right) .
$$

Then

$$
\operatorname{pi-hom}(D, H)=\operatorname{pi-hom}\left(D, H^{\prime}\right) \text {. }
$$

Lemma 3.9. Let $D \in \mathcal{D}_{k}$, and let $H, H^{\prime}$ be graphs. Suppose that for all $G \supseteq F^{D}$ with $V(G)=V(D)$ and $\left(G, T^{D}\right) \in \mathcal{D}_{k}$ we have

$$
\operatorname{pi-hom}\left(\left(G, T^{D}\right), H\right)=\operatorname{pi-hom}\left(\left(G, T^{D}\right), H^{\prime}\right) .
$$

Then

$$
\operatorname{pp}-\operatorname{hom}(D, H)=\operatorname{pp}-\operatorname{hom}\left(D, H^{\prime}\right) .
$$

Proof. Let $D=(F, T)$. Let $\mathcal{G}$ be the set of all $G \supseteq F$ such that $V(G)=V(F)$ and $T$ is an elimination tree of $G$. In particular, $F \in \mathcal{G}$. Let $G_{1}, \ldots, G_{m}$ be an enumeration of $\mathcal{G}$ such that $G_{1}=F$ and $\left|E\left(G_{i}\right)\right| \leq\left|E\left(G_{j}\right)\right|$ for $i \leq j$. Let $A \in \mathbb{R}^{m \times m}$ be the matrix 
with entries $A_{i j}:=1$ if $G_{i} \subseteq G_{j}$ and $A_{i j}=0$ otherwise. Then $A$ is an upper triangular matrix with diagonal entries $A_{i i}=1$ for all $i$. This implies that $A$ is invertible.

Let $\boldsymbol{c}=\left(c_{1}, \ldots, c_{m}\right)^{T}$ be the vector with entries $c_{i}:=\operatorname{pi-hom}\left(\left(G_{i}, T\right), H\right)$, and let $\boldsymbol{b}=\left(b_{1}, \ldots, b_{m}\right)^{T}$ be the vector with entries $b_{i}:=$ pp-hom $\left(\left(G_{i}, T\right), H\right)$. By Corollary 3.7, we have $\boldsymbol{c}=A \boldsymbol{b}$, and since $A$ is invertible, $\boldsymbol{b}=A^{-1} \boldsymbol{c}$.

Now let Let $\boldsymbol{c}^{\prime}=\left(c_{1}^{\prime}, \ldots, c_{m}^{\prime}\right)^{T}$ be the vector with entries $c_{i}^{\prime}:=\operatorname{pi-hom}\left(\left(G_{i}, T\right), H^{\prime}\right)$, and let $\boldsymbol{b}^{\prime}=\left(b_{1}^{\prime}, \ldots, b_{m}^{\prime}\right)^{T}$ be the vector with entries $b_{i}^{\prime}:=\operatorname{pp-hom}\left(\left(G_{i}, T\right), H^{\prime}\right)$. Then $\boldsymbol{b}^{\prime}=A^{-1} \boldsymbol{c}^{\prime}$.

By the assumption of the lemma, we have $\boldsymbol{c}=\boldsymbol{c}^{\prime}$. Thus $\boldsymbol{b}=\boldsymbol{b}^{\prime}$. In particular,

$$
\operatorname{pi-hom}(D, H)=b_{1}=b_{1}^{\prime}=\operatorname{pi}-\operatorname{hom}\left(D, H^{\prime}\right) .
$$

Theorem 3.10. For all $k \geq 0$ and all graphs $G, G^{\prime}$, the following are equivalent.

(i) For all $F \in \mathcal{T D}_{k}$,

$$
\operatorname{hom}(F, G)=\operatorname{hom}\left(F, G^{\prime}\right) .
$$

(ii) For all $D \in \mathcal{D}_{k}$,

$$
\operatorname{pi-hom}(D, G)=\operatorname{pi-hom}\left(D, G^{\prime}\right)
$$

(iii) For all $D \in \mathcal{D}_{k}$,

$$
\operatorname{pp}-\operatorname{hom}(D, G)=\operatorname{pp}-\operatorname{hom}\left(D, G^{\prime}\right) \text {. }
$$

Proof. The implication (i) $\Longrightarrow$ (ii) follows from Lemma 3.5.

As for all connected $F \in \mathcal{T}_{\mathcal{D}}^{c}$ there is an elimination tree $T$ such that $(F, T) \in \mathcal{D}_{k}$, it follows from Corollary 3.4 that (ii) implies $\operatorname{hom}(F, G)=\operatorname{hom}\left(F, G^{\prime}\right)$ for all $F \in \mathcal{T} \mathcal{D}_{k}^{c}$. But we have observed earlier that this implies $\operatorname{hom}(F, G)=\operatorname{hom}\left(F, G^{\prime}\right)$ for all $F \in \mathcal{T} \mathcal{D}_{k}$.

The equivalence between (ii) and (iii) follows from Lemma 3.9 and Corollary 3.8 .

\section{Playing the Game}

In this section, we will connect the numbers of past-preserving homomorphisms to the bijective pebble game and use this to prove our main theorem. We start with a technical lemma that we need for our interpolation arguments later 4

Lemma 4.1. Let $\boldsymbol{a}_{1}, \ldots, \boldsymbol{a}_{\ell} \in \mathbb{N}^{m}$, where $\boldsymbol{a}_{i}=\left(a_{i 1}, \ldots, a_{i m}\right)$, be mutually distinct vectors with positive entries. For every $i \in[\ell]$ and every $\boldsymbol{d}=\left(d_{1}, \ldots, d_{m}\right) \in \mathbb{N}^{m}$, let $\boldsymbol{a}_{i}^{(\boldsymbol{d})}:=\prod_{j=1}^{m} a_{i j}^{d_{j}}$.

Then there is a $\boldsymbol{d}=\left(d_{1}, \ldots, d_{m}\right) \in \mathbb{N}^{m}$ such that $1 \leq d_{i} \leq \ell^{2}$ for all $i \in[m]$ and $\boldsymbol{a}_{1}^{(\boldsymbol{d})}, \ldots, \boldsymbol{a}_{\ell}^{(\boldsymbol{d})}$ are mutually distinct.

Proof. The proof is by induction on $m$. The case $m=1$ is trivial, we can simply choose $d_{1}=1$. For the inductive step $m-1$ to $m$, let $\boldsymbol{a}_{i}^{\prime}:=\left(a_{i 1}, \ldots, a_{i(m-1)}\right)$. Let $P_{1}, \ldots, P_{\ell^{\prime}}$ be the partition of $[\ell]$ such that $\boldsymbol{a}_{i}^{\prime}=\boldsymbol{a}_{j}^{\prime}$ if and only if $i, j \in P_{p}$ for some $p \in\left[\ell^{\prime}\right.$.

\footnotetext{
${ }^{4}$ We are convinced that this lemma is known to many other researchers, but lacking a reference, we decided to include a proof.
} 
For $p \in\left[\ell^{\prime}\right]$, let $\boldsymbol{b}_{p}:=\boldsymbol{a}_{i}^{\prime}$ for $i \in P_{p}$. By the induction hypothesis, there is a vector $\boldsymbol{d}^{\prime}:=\left(d_{1}, \ldots, d_{m-1}\right)$ such that $1 \leq d_{i} \leq\left(\ell^{\prime}\right)^{2} \leq \ell^{2}$ for all $i \in[m-1]$ and the numbers $\boldsymbol{b}_{1}^{\left(\boldsymbol{d}^{\prime}\right)}, \ldots, \boldsymbol{b}_{\ell}^{\left(\boldsymbol{d}^{\prime}\right)}$ are mutually distinct. In the following, we keep $d_{1}, \ldots, d_{m-1}$ fixed and try to find a $d_{m}$ such that $\boldsymbol{d}=\left(d_{1}, \ldots, d_{m}\right)$ satisfies the assertion of the lemma.

Observe that for $d_{m} \geq 1$ and $i, j \in[\ell]$, if $i, j \in P_{p}$ for some $p$ then $\boldsymbol{a}_{i}^{\prime}=\boldsymbol{a}_{j}^{\prime}$ and $a_{i m} \neq a_{j m}$ and thus

$$
\boldsymbol{a}_{i}^{(\boldsymbol{d})}=\boldsymbol{b}_{p}^{\left(\boldsymbol{d}^{\prime}\right)} a_{i m}^{d_{m}} \neq \boldsymbol{b}_{p}^{\left(\boldsymbol{d}^{\prime}\right)} a_{j m}^{d m}=\boldsymbol{a}_{j}^{(\boldsymbol{d})} .
$$

For $i \in P_{p}, j \in P_{q}$ with $p \neq q$ we have

$$
\boldsymbol{a}_{i}^{(\boldsymbol{d})}=\boldsymbol{a}_{j}^{(\boldsymbol{d})} \Longleftrightarrow \frac{\boldsymbol{b}_{p}^{\left(\boldsymbol{d}^{\prime}\right)}}{\boldsymbol{b}_{q}^{\left(\boldsymbol{d}^{\prime}\right)}}=\frac{a_{j m}^{d_{m}}}{a_{i m}^{d_{m}}}
$$

If $a_{i m}=a_{j m}$, there is no such $d_{m}$, because $\frac{\boldsymbol{b}_{p}^{\left(\boldsymbol{d}^{\prime}\right)}}{\boldsymbol{b}_{q}^{\left(\boldsymbol{d}^{\prime}\right)}} \neq 1$. If $a_{i m} \neq a_{j m}$, there is at most one such $d_{m}$. Overall, for all distinct $i, j \in[\ell]$ there is at most one $d_{m} \geq 1$ such that $\boldsymbol{a}_{i}^{(\boldsymbol{d})}=\boldsymbol{a}_{j}^{(\boldsymbol{d})}$. Thus by the pigeonhole principle, there is a $d_{m} \leq\left(\begin{array}{l}\ell \\ 2\end{array}\right)+1 \leq \ell^{2}$ such that $\boldsymbol{a}_{i}^{(\boldsymbol{d})} \neq \boldsymbol{a}_{j}^{(\boldsymbol{d})}$ for all distinct $i, j$.

Let $D_{1}, \ldots, D_{m} \in \mathcal{D}$ such that the roots $r_{i}:=r^{D_{i}}$ all have the same colour, that is, $\gamma^{D_{i}}\left(r_{i}\right)=\gamma^{D_{j}}\left(r_{j}\right)=: c$ for all $i, j \in[m]$. We say that $D_{1}, \ldots, D_{m}$ are compatible. The rooted sum of $D_{1}, \ldots, D_{m}$ is the pair $D=(F, T)$ where $F$ is the graph obtained from the disjoint union of the $F^{D_{i}}$ by identifying the roots $r_{1}, \ldots, r_{m}$ and $T$ is the tree obtained from the disjoint union of the trees $T^{D_{1}}, \ldots, T^{D_{m}}$ by identifying their roots. We write $D=\bigoplus_{i=1}^{m} D_{i}$ to express that $D$ is the rooted sum of the $D_{i}$. For $d \geq 1$, we write $D=d \odot D^{\prime}$ to express that $D$ is the rooted sum of $d$ disjoint copies of $D^{\prime}$. We combine these notations, writing

$$
D=\bigoplus_{i=1}^{m} d_{i} \odot D_{i}
$$

to express that $D$ is the rooted sum of $d_{i}$ disjoint copies of $D_{i}$ for each $i \in[m]$. For every set $\mathcal{F} \subseteq \mathcal{D}$ we let $\mathcal{F}^{\oplus}$ denote the set of all rooted sums of elements of $\mathcal{F}$.

Recall that for a $D \in \mathcal{D}$, a graph $G$, and vertices $u \in V(D), v \in V(G)$, by pp-hom $(D, G ; u \mapsto$ $v$ ) we denote the number of past-preserving homomorphisms $h: D \rightarrow G$ with $h(u)=v$. Observe that if $D=\bigoplus_{i=1}^{m} D_{i}$ for $D_{i} \in \mathcal{D}_{k}$, then $D \in \mathcal{D}_{k}$ and for all graphs $G$ and vertices $v \in V(G)$ we have

$$
\operatorname{pp-hom}\left(D, G ; r^{D} \mapsto v\right)=\prod_{i=1}^{m} \mathrm{pp}-\operatorname{hom}\left(D_{i}, G ; r^{D_{i}} \mapsto v\right) .
$$

Lemma 4.2. Let $\mathcal{F} \subseteq \mathcal{D}$. Let $G, G^{\prime}$ be graphs such that $|G|=\left|G^{\prime}\right|$ and

$$
\operatorname{pp}-\operatorname{hom}(D, G)=\operatorname{pp}-\operatorname{hom}\left(D, G^{\prime}\right)
$$


for all $D \in \mathcal{F}^{\oplus}$. Then there is a bijection $f: V(G) \rightarrow V\left(G^{\prime}\right)$ such that

$$
\operatorname{pp}-\operatorname{hom}\left(D, G ; r^{D} \mapsto v\right)=\operatorname{pp}-\operatorname{hom}\left(D, G^{\prime} ; r^{D} \mapsto f(v)\right)
$$

for all $D \in \mathcal{F}$.

Proof. Let $n:=|G|=\left|G^{\prime}\right|$. Without loss of generality, we assume that $V(G) \cap V\left(G^{\prime}\right)=\emptyset$. We define an equivalence relation $\sim$ on $V(G) \cup V\left(G^{\prime}\right)$ as follows: for $X, Y \in\left\{G, G^{\prime}\right\}$ and $x \in V(X), y \in V(Y)$, we let $x \sim y$ if and only if for all $D \in \mathcal{F}$,

$$
\operatorname{pp-hom}\left(D, X ; r^{D} \mapsto x\right)=\operatorname{pp}-\operatorname{hom}\left(D, Y ; r^{D} \mapsto y\right) .
$$

Let $K_{1}, \ldots, K_{\ell}$ be the $\sim$-equivalence classes. For every $i \in[\ell]$, let $p_{i}:=\left|K_{i} \cap V(G)\right|$ and $p_{i}^{\prime}:=\left|K_{i} \cap V\left(G^{\prime}\right)\right|$, and let $\boldsymbol{p}=\left(p_{1}, \ldots, p_{\ell}\right)$ and $\boldsymbol{p}^{\prime}=\left(p_{1}^{\prime}, \ldots, p_{\ell}^{\prime}\right)$.

The assertion of the lemma is an immediate consequence of the following claim.

Claim 1.

$$
p=p^{\prime}
$$

Proof. Without loss of generality, we assume that $\mathcal{F}$ is finite. If it is not, for all distinct $i, j \in[\ell]$ we pick a $D_{i j} \in \mathcal{F}$ such that for $x \in K_{i}, y \in K_{j}$ and $X, Y \in\left\{G, G^{\prime}\right\}$ with $x \in V(X), y \in V(Y)$ it holds that

$$
\operatorname{pp}-\operatorname{hom}\left(D_{i j}, X ; r^{D_{i j}} \mapsto x\right) \neq \operatorname{pp}-\operatorname{hom}\left(D_{i j}, Y ; r^{D_{i j}} \mapsto y\right),
$$

and we restrict our attention to the finite class of all these $D_{i j}$ without changing the equivalence relation $\sim$.

Say, $\mathcal{F}=\left\{D_{1}, \ldots, D_{m}\right\}$, and for every $i \in[m]$, let $r_{i}:=r^{D_{i}}$. Then for all $X, Y \in$ $\left\{G, G^{\prime}\right\}$ and $x \in V(X), y \in V(Y)$ we have $x \sim y$ if and only if

$$
\forall j \in[m]: \operatorname{pp}-\operatorname{hom}\left(D_{j}, X ; r_{j} \mapsto x\right)=\operatorname{pp}-\operatorname{hom}\left(D_{j}, Y ; r_{j} \mapsto y\right) .
$$

For $i \in[\ell]$, let $a_{i j}:=\operatorname{pp}-\operatorname{hom}\left(D_{j}, X ; r_{j} \mapsto x\right)$ for all $X \in\left\{G, G^{\prime}\right\}, x \in K_{i} \cap V(X)$. Let $\boldsymbol{a}_{i}:=\left(a_{i 1}, \ldots, a_{i m}\right)$ and $\boldsymbol{a}^{j}=\left(a_{1 j}, \ldots, a_{\ell j}\right)^{T}$. Thus the $\boldsymbol{a}_{i}$ are the rows and the $\boldsymbol{a}^{j}$ the columns of the $(\ell \times m)$-matrix with entries $a_{i j}$. Observe that the rows $\boldsymbol{a}_{i}$ are mutually distinct.

For every $j \in[m]$ we have

$$
\begin{aligned}
\operatorname{pp-hom}\left(D_{j}, G\right) & =\sum_{v \in V(G)} \operatorname{pp-hom}\left(D_{j}, G ; r_{j} \mapsto v\right) \\
& =\sum_{i=1}^{\ell} p_{i} a_{i j} \\
& =\left\langle\boldsymbol{p}, \boldsymbol{a}^{j}\right\rangle .
\end{aligned}
$$

and similarly

$$
\operatorname{pp}-\operatorname{hom}\left(D_{j}, G^{\prime}\right)=\left\langle\boldsymbol{p}^{\prime}, \boldsymbol{a}^{j}\right\rangle \text {. }
$$


Here $\langle\boldsymbol{x}, \boldsymbol{y}\rangle=\sum_{i} x_{i} y_{i}$ denotes the standard inner product of vectors $\boldsymbol{x}, \boldsymbol{y}$.

Let us call a set $J \subseteq[m]$ compatible if all $D_{j}$ for $j \in J$ are compatible (that is, their roots have the same colour). The support of a vector $\boldsymbol{d}=\left(d_{1}, \ldots, d_{m}\right)$ is the set $\operatorname{supp}(\boldsymbol{d}):=\left\{i \in[m] \mid d_{i} \neq 0\right\}$, and we call $\boldsymbol{d}$ compatible if its support is compatible. For every compatible nonzero vector $\boldsymbol{d}=\left(d_{1}, \ldots, d_{m}\right) \in \mathbb{N}^{m}$ we let

$$
D^{(\boldsymbol{d})}:=\bigoplus_{j \in \operatorname{supp}(\boldsymbol{d})} d_{j} \odot D_{j} .
$$

Then $D^{(\boldsymbol{d})} \in \mathcal{F}^{\oplus}$. We denote the root of $D^{(\boldsymbol{d})}$ by $r^{(\boldsymbol{d})}$. By (4.A) , for every $X \in\left\{G, G^{\prime}\right\}$ and $x \in V(X)$ we have

$$
\operatorname{pp-hom}\left(D^{(\boldsymbol{d})}, X ; r^{(\boldsymbol{d})} \mapsto x\right)=\prod_{j=1}^{m} \operatorname{pp-hom}\left(D_{j}, X ; r_{j} \mapsto x\right)^{d_{j}} .
$$

Thus for every $i \in[\ell], X \in\{G, H\}$, and $x \in K_{i} \cap V(X)$ we have

$$
\operatorname{pp-hom}\left(D^{(\boldsymbol{d})}, X ; r^{(\boldsymbol{d})} \mapsto x\right)=\prod_{j=1}^{m} a_{i j}^{d_{j}}=: \boldsymbol{a}_{i}^{(\boldsymbol{d})} .
$$

Let $\boldsymbol{a}^{(\boldsymbol{d})}=\left(\boldsymbol{a}_{1}^{(d)}, \ldots, \boldsymbol{a}_{\ell}^{(d)}\right)^{T}$. Note that with this notation, $\boldsymbol{a}^{j}=\boldsymbol{a}^{\left(\boldsymbol{e}_{j}\right)}$, where $\boldsymbol{e}_{j}$ denotes the $j$ th unit vector. Then

$$
\begin{aligned}
\operatorname{pp}-\operatorname{hom}\left(D^{(\boldsymbol{d})}, G\right) & =\sum_{v \in V(G)} \operatorname{pp-hom}\left(D^{(\boldsymbol{d})}, G ; r^{(d)} \mapsto v\right) \\
& =\sum_{i=1}^{\ell} p_{i} \boldsymbol{a}_{i}^{(d)} \\
& =\left\langle\boldsymbol{p}, \boldsymbol{a}^{(d)}\right\rangle .
\end{aligned}
$$

and similarly

$$
\operatorname{pp}-\operatorname{hom}\left(D^{(\boldsymbol{d})}, G^{\prime}\right)=\left\langle\boldsymbol{p}^{\prime}, \boldsymbol{a}^{(\boldsymbol{d})}\right\rangle .
$$

For all $j$, let $b^{(\boldsymbol{d})}:=\operatorname{pp}-\operatorname{hom}\left(D^{(\boldsymbol{d})}, G\right)$. Since $D^{(\boldsymbol{d})} \in \mathcal{F}^{\oplus}$, by 4 .B. , we have $b^{(\boldsymbol{d})}=$ $\operatorname{pp}-\operatorname{hom}\left(D^{(\boldsymbol{d})}, G^{\prime}\right)$. Then

$$
b^{(\boldsymbol{d})}=\left\langle\boldsymbol{p}, \boldsymbol{a}^{(\boldsymbol{d})}\right\rangle=\left\langle\boldsymbol{p}^{\prime}, \boldsymbol{a}^{(\boldsymbol{d})}\right\rangle .
$$

Since $0^{0}=1$ and $0^{d}=0$ for $d \geq 1$, for every $i \in[\ell]$ and every $\boldsymbol{d} \in \mathbb{N}^{m}$ we have

$$
\boldsymbol{a}_{i}^{(\boldsymbol{d})} \neq 0 \Longleftrightarrow \operatorname{supp}(\boldsymbol{d}) \subseteq \operatorname{supp}\left(\boldsymbol{a}_{i}\right)
$$

Suppose for contradiction that $\boldsymbol{p} \neq \boldsymbol{p}^{\prime}$. Choose $i_{0} \in[\ell]$ such that $p_{i_{0}} \neq p_{i_{0}}^{\prime}$ and, subject to this condition, $S:=\operatorname{supp}\left(\boldsymbol{a}_{i_{0}}\right)$ is inclusionwise maximal. 
Suppose first that $S=\emptyset$. Then $\boldsymbol{a}_{i_{0}}=\mathbf{0}$, and as the $\boldsymbol{a}_{i}$ are mutually distinct, $\boldsymbol{a}_{i} \neq \mathbf{0}$ and therefore $\operatorname{supp}\left(\boldsymbol{a}_{i}\right) \neq \emptyset$ for $i \neq i_{0}$. By the maximality of $S$, this implies $p_{i}=p_{i}^{\prime}$ for all $i \neq i_{0}$. Hence $p_{i_{0}}=n-\sum_{i \neq i_{0}} p_{i}=n-\sum_{i \neq i_{0}} p_{i}^{\prime}=p_{i_{0}}^{\prime}$, which is a contradiction. It follows that $S \neq \emptyset$.

Let $I=\left\{i \in[\ell] \mid \operatorname{supp}\left(\boldsymbol{a}_{i}\right)=S\right\}$. For every $i \in I$, let $\widehat{\boldsymbol{a}}_{i}:=\left(a_{i j} \mid j \in S\right)$. Then the vectors $\widehat{\boldsymbol{a}}_{i}$ have only positive entries, and they are mutually distinct, because the $\boldsymbol{a}_{i}$ are mutually distinct. By Lemma 4.1, there is a vector $\widehat{\boldsymbol{d}}=\left(\widehat{d}_{j} \mid j \in S\right)$ such that the numbers $\widehat{\boldsymbol{a}}_{i}^{(\widehat{\boldsymbol{d}})}$ for $i \in I$ are mutually distinct. Let $\boldsymbol{d}=\left(d_{1}, \ldots, d_{m}\right)$ with $d_{j}=\widehat{d}_{j}$ for $j \in S$ and $d_{j}=0$ otherwise. Then the numbers $\boldsymbol{a}_{i}^{(\boldsymbol{d})}$ for $i \in I$ are mutually distinct.

Observe that for every $j \in \mathbb{N}$ we have $\boldsymbol{a}_{i}^{(j \boldsymbol{d})}=\left(\boldsymbol{a}_{i}^{(\boldsymbol{d})}\right)^{j}$, where $j \boldsymbol{d}=\left(j d_{1}, \ldots, j d_{m}\right)$. Let $A$ be the $|I| \times|I|$-matrix with entries $A_{i j}:=\boldsymbol{a}_{i}^{(j \boldsymbol{d})}$ (for convenience, we take row indices from the set $I$ and column indices from $\{1, \ldots,|I|\}) . A$ is a Vandermonde matrix and thus invertible.

Let $\boldsymbol{p}_{I}:=\left(p_{i} \mid i \in I\right)$ and $\boldsymbol{p}_{I}^{\prime}:=\left(p_{i}^{\prime} \mid i \in I\right)$ be the restrictions of $\boldsymbol{p}$ and $\boldsymbol{p}^{\prime}$ to $I$. For every $j$, the $j$ th entry of $\boldsymbol{p}_{I} \cdot A$ is

$$
\begin{aligned}
\sum_{i \in I} p_{i} \boldsymbol{a}_{i}^{(j \boldsymbol{d})} & =\sum_{i=1}^{\ell} p_{i} \boldsymbol{a}_{i}^{(j \boldsymbol{d})}-\sum_{\substack{i \in[\ell] \\
S \nsubseteq \operatorname{supp}_{\left(\boldsymbol{a}_{i}\right)}}} p_{i} \boldsymbol{a}_{i}^{(j \boldsymbol{d})}-\sum_{\substack{i \in[\ell] \\
S \subset \operatorname{supp}\left(\boldsymbol{a}_{i}\right)}} p_{i} \boldsymbol{a}_{i}^{(j \boldsymbol{d})} \\
& =\left\langle\boldsymbol{p}, \boldsymbol{a}^{(j \boldsymbol{d})}\right\rangle-\sum_{\substack{i \in[\ell] \\
S \subset \operatorname{supp}\left(\boldsymbol{a}_{i}\right)}} p_{i} \boldsymbol{a}_{i}^{(j \boldsymbol{d})},
\end{aligned}
$$

because by (4.F) we have $a_{i}^{(j \boldsymbol{d})}=0$ if $S=\operatorname{supp}(j \boldsymbol{d}) \nsubseteq \operatorname{supp}\left(\boldsymbol{a}_{i}\right)$. Similarly, the $j$ th entry of $\boldsymbol{p}_{I}^{\prime} \cdot A$ is

$$
\sum_{i \in I} p_{i}^{\prime} \boldsymbol{a}_{i}^{(j \boldsymbol{d})}=\left\langle\boldsymbol{p}^{\prime}, \boldsymbol{a}^{(j \boldsymbol{d})}\right\rangle-\sum_{\substack{i \in[\ell] \\ S \subset \operatorname{supp}\left(\boldsymbol{a}_{i}\right)}} p_{i}^{\prime} a_{i}^{(j \boldsymbol{d})} .
$$

By the maximality of $S$, for all $i$ with $S \subset \operatorname{supp}\left(\boldsymbol{a}_{i}\right)$ we have $p_{i}=p_{i}^{\prime}$. Thus

$$
\sum_{\substack{i \in[\ell] \\ S \subset \operatorname{supp}\left(\boldsymbol{a}_{i}\right)}} p_{i} \boldsymbol{a}_{i}^{(j \boldsymbol{d})}=\sum_{\substack{i \in[\ell] \\ S \subset \operatorname{supp}\left(\boldsymbol{a}_{i}\right)}} p_{i}^{\prime} \boldsymbol{a}_{i}^{(j \boldsymbol{d})},
$$

and therefore, by (4.E),

$$
\sum_{i \in I} p_{i} \boldsymbol{a}_{i}^{(j \boldsymbol{d})}=\sum_{i \in I} p_{i}^{\prime} \boldsymbol{a}_{i}^{(j \boldsymbol{d})}
$$

Since this holds for all $j$, we have $\boldsymbol{p}_{I} \cdot A=\boldsymbol{p}_{I}^{\prime} \cdot A$. As $A$ is invertible, it follows that $\boldsymbol{p}_{I}=\boldsymbol{p}_{I}^{\prime}$ and, in particular, $p_{i_{0}}=p_{i_{0}}^{\prime}$. This is a contradiction.

Remark 4.3. The proof of the lemma actually shows that (4.B) only needs to be satisfied for rooted sums of at most $m \ell^{2}$ graphs from $\mathcal{F}$, where $m:=|\mathcal{F}|$, and $\ell$ is the number of equivalence classes of the relation $\sim$. (Recall the definition of $\sim$ from (4.C).) Note that we always have $\ell \leq n=|G|$. 
Corollary 4.4. Let $G, G^{\prime}$ be graphs such that for all $D \in \mathcal{D}_{k}$,

$$
\operatorname{pp}-\operatorname{hom}(D, G)=\operatorname{pp}-\operatorname{hom}\left(D, G^{\prime}\right) .
$$

Then there is a bijection $f: V(G) \rightarrow V\left(G^{\prime}\right)$ such that for all $D \in \mathcal{D}_{k}$,

$$
\operatorname{pp}-\operatorname{hom}\left(D, G ; r^{D} \mapsto v\right)=\mathrm{pp}-\operatorname{hom}\left(D, G^{\prime} ; r^{D} \mapsto f(v)\right) .
$$

Proof. This follows immediately from Lemma 4.2, noting that $\mathcal{D}_{k}^{\oplus}=\mathcal{D}_{k}$ and that (4.G) for all $D \in \mathcal{D}_{k}$ implies that $|G|=|H|$.

\subsection{Proof of the Main Theorem}

For the inductive proof, the following construction is useful. Let $G$ be a graph and $v \in V(G)$. We let $G \imath v$ be the graph with vertex set $V(G \succ v):=V(G) \backslash\{v\}$, edge set $E(G \prec v):=\left\{w w^{\prime} \in E(G) \mid w, w^{\prime} \in V(G) \backslash\{v\}\right\}$, and colouring defined by

$$
\gamma^{G l v}(w):= \begin{cases}\left(\gamma^{G}(w), 1\right) & \text { if } v w \in E(G), \\ \left(\gamma^{G}(w), 0\right) & \text { if } v w \notin E(G) .\end{cases}
$$

Lemma 4.5. Let $k \geq 0$, and let $G, G^{\prime}$ be graphs and $v \in V(G), v^{\prime} \in V\left(G^{\prime}\right)$ such that $|G|=\left|G^{\prime}\right| \geq 2$ and $\gamma^{G}(v)=\gamma^{G^{\prime}}\left(v^{\prime}\right)$. Then the following are equivalent.

(i) Duplicator has a winning strategy for the k-round bijective pebble game on $G, G^{\prime}$ with initial position $\left(v, v^{\prime}\right)$.

(ii) Duplicator has a winning strategy for the k-round bijective pebble game on $G \nmid v$, $G^{\prime} \prec v^{\prime}$.

Proof. Straightforward.

The next lemma is the last significant step of the proof of our main theorem. After that, we only need to pull things together to complete the proof.

Lemma 4.6. Let $k \geq 1$, and let $G, G^{\prime}$ be graphs of the same order. Then the following are equivalent.

(i) For all $D \in \mathcal{D}_{k}$,

$$
\operatorname{pp}-\operatorname{hom}(D, G)=\operatorname{pp}-\operatorname{hom}\left(D, G^{\prime}\right)
$$

(ii) Duplicator has a winning strategy for the $k$-round bijective pebble game on $G, G^{\prime}$.

Proof. We first prove (i) $\Longrightarrow$ (ii). The proof is by induction on $k$.

For the base case $k=1$, suppose that pp-hom $(D, G)=\operatorname{pp}-\operatorname{hom}\left(D, G^{\prime}\right)$ for all $D \in \mathcal{D}_{1}$. By Corollary 4.4, there is a bijection $f: V(G) \rightarrow V\left(G^{\prime}\right)$ such that for all $D \in \mathcal{D}_{1}$ and $v \in V(G)$,

$$
\operatorname{pp}-\operatorname{hom}\left(D, G ; r^{D} \mapsto v\right)=\operatorname{pp}-\operatorname{hom}\left(D, G^{\prime} ; r^{D} \mapsto f(v)\right) .
$$


This implies $\gamma^{G}(v)=\gamma^{G^{\prime}}(f(v))$. Duplicator picks $f$ in the first (and only) round of the game and wins.

For the inductive step $k \rightarrow k+1$, let $G, G^{\prime}$ be graphs of the same order such that $\operatorname{pp}-\operatorname{hom}(D, G)=\operatorname{pp}-\operatorname{hom}\left(D, G^{\prime}\right)$ for all $D \in \mathcal{D}_{k+1}$. If $|G|=\left|G^{\prime}\right|=1$, then pp-hom $(D, G)=\operatorname{pp-hom}\left(D, G^{\prime}\right)$ for all $D \in \mathcal{D}_{1}$ implies that the graphs are isomorphic (their unique vertices have the same colour). Thus we may further assume that $|G|=\left|G^{\prime}\right| \geq 2$.

By Corollary 4.4, there is a bijection $f: V(G) \rightarrow V\left(G^{\prime}\right)$ such that for all $D \in \mathcal{D}_{k+1}$ and $v \in V(G)$,

$$
\operatorname{pp}-\operatorname{hom}\left(D, G ; r^{D} \mapsto v\right)=\operatorname{pp}-\operatorname{hom}\left(D, G^{\prime} ; r^{D} \mapsto f(v)\right) .
$$

In the first round of the game, Duplicator picks this bijection $f$. Say, Spoiler picks $v \in V(G)$. Let $v^{\prime}:=f(v)$. Note that (4.H) implies $\gamma^{G}(v)=\gamma^{G^{\prime}}\left(v^{\prime}\right)$. Let $H:=G$ ? $v$ and $H^{\prime}:=G^{\prime}\left\{v^{\prime}\right.$. We need to prove that Duplicator has a winning strategy for the remaining $k$-round bijective pebble game on $G, G^{\prime}$ with initial position $\left(v, v^{\prime}\right)$. By Lemma 4.5, it suffices to prove that Duplicator has a winning strategy for the $k$-round bijective pebble game on $H, H^{\prime}$. This follows immediately from the induction hypothesis and the following claim.

Claim 1. For all $D \in \mathcal{D}_{k}$,

$$
\operatorname{pp}-\operatorname{hom}(D, H)=\operatorname{pp}-\operatorname{hom}\left(D, H^{\prime}\right) .
$$

Proof. Let $D=(F, T) \in \mathcal{D}_{k}$. We may assume that all vertices $u \in V(D)$ have a colour of the form $(c, i)$ where $i \in\{0,1\}$. Otherwise, $\operatorname{pp-hom}(D, H)=\operatorname{pp}-\operatorname{hom}\left(D, H^{\prime}\right)=0$.

We define a graph $F^{+}$and a tree $T^{+}$as follows. We take a fresh vertex $r^{+}$and let $V\left(F^{+}\right):=V(F) \cup\left\{r^{+}\right\}$,

$$
\begin{aligned}
E\left(F^{+}\right):= & E(F) \cup \\
& \left\{r^{+} u \mid u \in V(F) \text { with } \gamma^{F}(u)=(c, 1) \text { for some } c\right\},
\end{aligned}
$$

and $\gamma^{F^{+}}\left(r^{+}\right):=\gamma^{G}(v)=\gamma^{G^{\prime}}\left(v^{\prime}\right)$ and $\gamma^{F^{+}}(u):=c$ for all $u \in V(F)$ with $\gamma^{F}(u)=(c, i)$ for some $i \in\{0,1\}$. We let $V\left(T^{+}\right):=V(T) \cup\left\{r^{+}\right\}$and

$$
\preceq^{T^{+}}:=\preceq^{T} \cup\left\{\left(r^{+}, u\right) \mid u \in V(T)\right\} .
$$

Then $r^{+}$is the root of $T^{+}$. Then $T^{+}$is an elimination tree of $F^{+}$of height $k+1$. Hence $D^{+}:=\left(F^{+}, T^{+}\right) \in \mathcal{D}_{k+1}$.

Observe that that there is a one-to-one correspondence between the past-preserving homomorphisms from $D^{+}$to $G$ mapping $r^{+}$to $v$ and the past-preserving homomorphisms from $D$ to $H 5$ Similarly, there is a one-to-one correspondence between the pastpreserving homomorphisms from $D^{+}$to $G^{\prime}$ mapping $r^{+}$to $v^{\prime}$ and the past-preserving

\footnotetext{
${ }^{5}$ Note that here we need the homomorphisms to be past preserving. The proof would break down if we worked with arbitrary homomorphisms, because a homomorphism from $D^{+}$to $G$ mapping $r^{+}$ to $v$ could also map vertices in $V\left(D^{+}\right) \backslash\left\{r^{+}\right\}=V(D)$ to $v$, and such a homomorphism would not correspond to a homomorphism from $D$ to $H$.
} 
homomorphisms from $D$ to $H^{\prime}$. Thus

$$
\begin{aligned}
\operatorname{pp}-\operatorname{hom}(D, H) & =\operatorname{pp}-\operatorname{hom}\left(D^{+}, G ; r^{+} \mapsto v\right), \\
\mathrm{pp}-\operatorname{hom}\left(D, H^{\prime}\right) & =\operatorname{pp}-\operatorname{hom}\left(D^{+}, G^{\prime} ; r^{+} \mapsto v^{\prime}\right) .
\end{aligned}
$$

By (4.H) applied to $D^{+}$, we have

$$
\operatorname{pp}-\operatorname{hom}\left(D^{+}, G ; r^{+} \mapsto v\right)=\operatorname{pp-hom}\left(D^{+}, G^{\prime} ; r^{+} \mapsto v^{\prime}\right) .
$$

$\operatorname{Thus} \operatorname{pp}-\operatorname{hom}(D, H)=\mathrm{pp}-\operatorname{hom}\left(D, H^{\prime}\right)$.

The proof of the converse direction (ii) $\Longrightarrow$ (i) is also by induction on $k$.

For the base case $k=1$, assume that Duplicator has a winning strategy for the 1move bijective pebble game on $G, G^{\prime}$. Then there is a bijection $f: V(G) \rightarrow V\left(G^{\prime}\right)$ such that $\gamma^{G}(v)=\gamma^{G^{\prime}}(f(v))$ for all $v \in V(G)$, which implies that for each colour $c$ the two graphs have the same numbers of vertices of colour $c$. This implies that pp-hom $(D, G)=\operatorname{pp-hom}\left(D, G^{\prime}\right)$ for all $D \in \mathcal{D}_{1}$.

For the inductive step $k \rightarrow k+1$, assume that Duplicator has a winning strategy for the $(k+1)$-round bijective pebble game on $G, G^{\prime}$. Without loss of generality we may assume that $|G|=\left|G^{\prime}\right| \geq 2$. Then, by Lemma 4.5, there is a bijection $f: V(G) \rightarrow V\left(G^{\prime}\right)$ such that for each $v \in V(G), \gamma^{G}(v)=\gamma^{G^{\prime}}(f(v))$ and Duplicator has a winning strategy for the $k$-round bijective pebble game on $G \imath v, G^{\prime} \prec f(v)$. By the induction hypothesis, this implies

$$
\operatorname{pp}-\operatorname{hom}(D, G \succ v)=\operatorname{pp}-\operatorname{hom}\left(D, G^{\prime} \prec f(v)\right)
$$

for all $D \in \mathcal{D}_{k}$.

Now let $\widehat{D} \in \mathcal{D}_{k+1}$ and $\widehat{r}:=r^{\widehat{D}}$. By deleting $\widehat{r}$ from $\widehat{D}$, we obtain a family $D_{1}, \ldots, D_{m} \in$ $\mathcal{D}_{k}$. For every $i \in[m]$, let $\widehat{D}_{i} \in \mathcal{D}_{k}$ be obtained from $D_{i}$ by recolouring the vertices as follows: for $u \in V\left(D_{i}\right)$, let

$$
\gamma^{\widehat{D}_{i}}(u):= \begin{cases}\left(\gamma^{D_{i}}(u), 1\right) & \text { if } \widehat{r} u \in E(\widehat{D}), \\ \left(\gamma^{D_{i}}(u), 0\right) & \text { otherwise. }\end{cases}
$$

The crucial observation is that for each $v \in V(G)$ with $\gamma^{G}(v)=\gamma^{D}(\widehat{r})$ we have

$$
\operatorname{pp}-\operatorname{hom}(\widehat{D}, G ; \widehat{r} \mapsto v)=\prod_{i=1}^{m} \mathrm{pp}-\operatorname{hom}\left(\widehat{D}_{i}, G \succ v\right)
$$

and similarly

$$
\operatorname{pp}-\operatorname{hom}\left(\widehat{D}, G^{\prime} ; \widehat{r} \mapsto f(v)\right)=\prod_{i=1}^{m} \operatorname{pp}-\operatorname{hom}\left(\widehat{D}_{i}, G^{\prime} \curlywedge f(v)\right) .
$$

By (4.I), this implies

$$
\operatorname{pp}-\operatorname{hom}(\widehat{D}, G ; \widehat{r} \mapsto v)=\operatorname{pp}-\operatorname{hom}\left(\widehat{D}, G^{\prime} ; \widehat{r} \mapsto f(v)\right) .
$$


Thus

$$
\begin{aligned}
\operatorname{pp}-\operatorname{hom}(\widehat{D}, G)= & \sum_{\substack{v \in V(G) \\
\gamma^{G}(v)=\gamma^{\widehat{D}}(\widehat{r})}} \operatorname{pp-hom}(\widehat{D}, G ; \widehat{r} \mapsto v) \\
= & \sum_{\substack{v \in V(G) \\
\gamma^{G}(v)=\gamma^{\widehat{D}}(\widehat{r})}} \operatorname{pp-hom}\left(\widehat{D}, G^{\prime} ; \widehat{r} \mapsto f(v)\right) \\
= & \sum_{\substack{v^{\prime} \in V\left(G^{\prime}\right) \\
\sigma^{\prime}\left(v^{\prime}\right)=\gamma^{\widehat{D}}(\widehat{r}) \\
=}} \operatorname{pp}-\operatorname{hom}\left(\widehat{D}, G^{\prime}\right) .
\end{aligned}
$$

Proof of Theorem 1.1. The theorem follows from the previous lemma combined with Lemma 2.2 (stating that winning strategies for Duplicator in the bijective pebble game establish equivalence in the logic) and Theorem 3.10 (stating the equivalence between homomorphism counts and past-preserving homomorphism counts), observing that for all $k \geq 1$, graphs $G, G^{\prime}$ of distinct orders are neither homomorphism-indistinguishable over the class $\mathcal{T} \mathcal{D}_{k}$ nor $\mathrm{C}_{k}$-equivalent.

\section{Discussion}

It is a consequence of our main theorem that every sentence $\varphi$ of the logic $C$ and other counting logics such as Kuske and Schweikardt's 25. FOCN $(\mathbf{P})$ is equivalent to an infinitary Boolean combination of expressions of $\eta_{F, m}$ stating that "there are exactly $m$ homomorphism from $F$ into the current graph", where $F \in \mathcal{T} \mathcal{D}_{k}$ for the quantifier rank $k$ of $\varphi$. Indeed, it follows immediately from Theorem 1.1 that every sentence $\varphi \in \mathrm{C}$ of quantifier rank $k$ is equivalent to

$$
\bigvee_{\begin{array}{c}
G \text { graph } \\
\text { such that } G=\varphi
\end{array}} \bigwedge_{F \in \mathcal{T} \mathcal{D}_{k}^{c}} \eta_{F, \operatorname{hom}(F, G)} .
$$

Observe that $\eta_{F, m}$ can be viewed as a sentence of the form $\exists^{=m} \boldsymbol{x} \alpha$, where $\boldsymbol{x}$ is a tuple of $|F|$ variables and $\alpha$ is a conjunction of atoms of the form $E\left(x_{i}, x_{j}\right)$ and $\gamma\left(x_{i}\right)=$ c. This gives us a normal form for C-sentences that is local and achieves some form of quantifier elimination (or, maybe more precisely, quantifier de-alternation). But of course the infinite disjunction and conjunctions are unpleasant. We can replace the infinite conjunctions by a finite one, ranging over a finite set $\mathcal{F}(G) \subseteq \mathcal{T D}_{k}^{c}$ that only depends on $G$. But there is no hope of avoiding the infinite disjunction.

\subsection{Graphs of Bounded Degree}

For graphs $G$ of bounded degree, we can improve our main theorem. We fix a set $\Gamma$ of colours and only consider graphs $G$ with $\operatorname{rg}\left(\gamma^{G}\right) \subseteq \Gamma$. 
Theorem 5.1. Let $k, d \geq 1$. Then there is a finite set $\mathcal{F}_{k, d} \subseteq \mathcal{T D}_{k}$, computable from $k, d$, such that for all graphs $G, G^{\prime}$ of maximum degree at most $d$ the following are equivalent.

(i) $G$ and $G^{\prime}$ are homomorphism-indistinguishable over $\mathcal{F}_{k, d}$.

(ii) $G$ and $G^{\prime}$ satisfy the same $\mathrm{C}_{k}$-sentences.

Proof (sketch). We only need to prove that the implication (i) $\Longrightarrow$ (ii) holds for a sufficiently large $\mathcal{F}_{k, d} \subseteq \mathcal{T} \mathcal{D}_{k}$.

Recall that connected graphs in $\mathcal{T} \mathcal{D}_{k}$ have radius at most $2^{k-1}-1$. Thus if two vertices $v, w$ in a graph $G$ have isomorphic neighbourhoods of radius $2^{k-1}-1$, then $\operatorname{hom}\left(D, G ; r^{D} \mapsto v\right)=\operatorname{hom}\left(D, G ; r^{D} \mapsto v^{\prime}\right)$ for all $D \in \mathcal{D}_{k}$. Note that the equality holds even though the graph $F^{D}$ is not necessarily connected, because on both sides of the equality we have the same graph $G$, and every connected component of $D$ that does not contain the root $r^{D}$ contributes to both sides of the equation in the same way.

In graphs of maximum degree at most $d$, the number of isomorphism types of neighbourhoods of radius $2^{k-1}-1$ is bounded in terms of $k$ and $d$. This means that there is only a bounded number of homomorphism counts $\operatorname{hom}\left(D, G ; r^{D} \mapsto v\right)$. Recall Remark 4.3. By what we have just observed, if both $G$ and $G^{\prime}$ are of maximum degree $d$, the number $\ell$ of equivalence classes is bounded in terms of $k, d$, and thus we only need to consider rooted sums of at most $f(k, d)$ graphs (for a suitable function $f$ ).

If we plug this into the inductive proof of the main theorem, we see that we only need to consider homomorphism counts from $g(k, d)$ graphs, for a suitable function $g$.

Note that this stronger version of the theorem leads to a slight improvement of the normal form (5.A) for graphs of maximum degree at most $d$ : independently of the disjunct $G$, we can restrict the conjunction to graphs $F$ from the finite set $\mathcal{F}_{k, d}$.

\subsection{Equivalence in First-Order Logic}

We may wonder if in Theorem 5.1 we really need the dependence of the set $\mathcal{F}_{k, d}$ on the maximum degree $d$. That is, we may ask if for every $k$ there is a finite set $\mathcal{F}_{k} \subseteq \mathcal{T} \mathcal{D}_{k}$ such that for all graphs $G, G^{\prime}$ if $G$ and $G^{\prime}$ are homomorphism-indistinguishable over $\mathcal{F}_{k}$ then they are $C_{k}$-equivalent. It is easy to see that this cannot be the case, essentially because the number of $\mathrm{C}_{k}$-equivalence classes is unbounded.

However, this is different for first-order logic FO: for every $k$ there are only finitely many $\mathrm{FO}_{k}$-equivalence classes, where $\mathrm{FO}_{k}$ denotes the fragment of $\mathrm{FO}$ consisting of all formulas of quantifier rank at most $k$. Thus it may be tempting to conjecture the following. Again, we fix a set $\Gamma$ of colours and only consider graphs $G$ with $\operatorname{rg}\left(\gamma^{G}\right) \subseteq \Gamma$.

Conjecture 5.2. Let $k \geq 1$. Then there is a finite set $\mathcal{F}_{k} \subseteq \mathcal{T D}_{k}$ such that for all graphs $G, G^{\prime}$, if $G$ and $G^{\prime}$ are homomorphism-indistinguishable over $\mathcal{F}_{k}$, then $G$ and $G^{\prime}$ satisfy the same $\mathrm{FO}_{k}$-sentences.

It would be really nice if this conjecture was true. For example, it would imply a parameterised version of Toda's theorem, settling a long-standing open problem in 


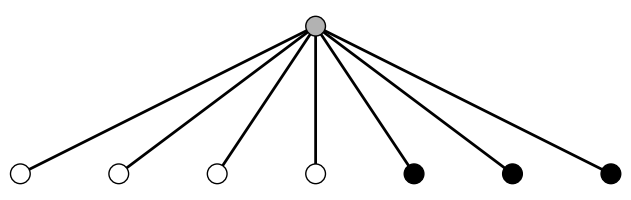

Figure 5.1. The star $S_{4,3}$

parameterised complexity theory [18]. Unfortunately, the conjecture is false already for $k=2$.

Example 5.3. Let us assume that $\Gamma=\{\circ, \bigcirc, \bullet\}$. For all $k, \ell \in \mathbb{N}$, we let $S_{p, q}$ be the star with a centre $r$ and tips $s_{1}, \ldots, s_{p}, t_{1}, \ldots, t_{q}$ such that $r$ is grey, the $s_{i}$ are white, and the $t_{j}$ are black (see Figure 5.1). Moreover, we let $W$ be the graph consisting of a single white vertex and $B$ the graph consisting of a single black vertex. Observe that $S_{0,0}, W, B \in \mathcal{T D}_{1}$ and $S_{p, q} \in \mathcal{T} \mathcal{D}_{2}$ for all $p, q \in \mathbb{N}$.

Let $\mathcal{S}$ be the class of all graphs that are finite disjoint unions of stars $S_{p, q}$ for $p, q \in \mathbb{N}$. For every graph $G \in \mathcal{S}$ and all $p, q \in \mathbb{N}$, let $a_{p, q}(G)$ be the number of copies of $S_{p, q}$ in $G$. Observe that for all $i, j, p, q \in \mathbb{N}$ we have

$$
\operatorname{hom}\left(S_{i, j}, S_{p, q}\right)=p^{i} q^{j} .
$$

Thus for $G \in \mathcal{S}$,

$$
\operatorname{hom}\left(S_{i, j}, G\right)=\sum_{p, q \in \mathbb{N}} p^{i} q^{j} a_{p, q}(G) .
$$

Moreover, $\operatorname{hom}(W, G)=\operatorname{hom}\left(S_{1,0}, G\right)=\sum_{p, q \in \mathbb{N}} p a_{p, q}(G)$ and $\operatorname{hom}(B, G)=\operatorname{hom}\left(S_{0,1}, G\right)=$ $\sum_{p, q \in \mathbb{N}} q a_{p, q}(G)$. Observe that $\operatorname{hom}(F, G)=0$ for all connected $F \in \mathcal{T} \mathcal{D}_{2} \backslash(\mathcal{S} \cup\{B, W\})$.

Suppose for contradiction that there is a finite $\mathcal{F} \subseteq \mathcal{T} \mathcal{D}_{2}$ such that for all graphs $G, G^{\prime}$, if $\operatorname{hom}(F, G)=\operatorname{hom}\left(F, G^{\prime}\right)$ for all $F \in \mathcal{F}$ then $G \equiv_{2}^{C} G^{\prime}$. Without loss of generality we assume that all $F \in \mathcal{F}$ are connected. We will only consider graphs $G, G^{\prime} \in \mathcal{S}$. Thus it suffices to consider $F \in \mathcal{F} \cap\left\{S_{i, j} \mid i, j \in \mathbb{N}\right\}$. Let $m:=\max \left\{j \mid S_{i, j} \in \mathcal{F}\right\}$.

Claim 1. There are vectors $\boldsymbol{a}=\left(a_{1}, \ldots, a_{m}\right)$, $\boldsymbol{a}^{\prime}=\left(a_{1}^{\prime}, \ldots, a_{m}^{\prime}\right) \in \mathbb{N}^{m}$ such that

(i) $\sum_{q=1}^{m} a_{q}<\sum_{q=1}^{m} a_{q}^{\prime}$,

(ii) $\sum_{q=1}^{m} q^{j} a_{q}=\sum_{q=1}^{m} q^{j} a_{q}^{\prime}$ for all $j \in[m]$.

Proof. Let $A \in \mathbb{Q}^{m \times m}$ be the matrix with entries $a_{i j}=j^{i-1}$. Then $A$ is a Vandermonde matrix and thus has full rank. Therefore, the equation $A \boldsymbol{x}=\boldsymbol{e}_{1}$, where $\boldsymbol{e}_{1}=(1,0, \ldots, 0)^{T} \in \mathbb{Q}^{m}$, has the rational solution $\boldsymbol{x}=A^{-1} \boldsymbol{e}_{1}$. Multiplying with a positive common denominator $c$ of the entries of $\boldsymbol{x}$, we obtain an integer solution $\boldsymbol{y}$ to the system $A \boldsymbol{y}=c \boldsymbol{e}_{1}$. We write $\boldsymbol{y}=\boldsymbol{a}^{\prime}-\boldsymbol{a}$ for two nonnegative integer vectors 
$\boldsymbol{a}^{\prime}=\left(a_{1}^{\prime}, \ldots, a_{n}^{\prime}\right), \boldsymbol{a}=\left(a_{1}, \ldots, a_{m}\right)$. The equation $A \boldsymbol{a}^{\prime}=c \boldsymbol{e}_{1}+A \boldsymbol{a}$ yields exactly the equations in the assertion of the claim.

We choose vectors $\boldsymbol{a}, \boldsymbol{a}^{\prime}$ according to the claim and let $a_{0}:=\sum_{p=1}^{m} a_{p}^{\prime}-\sum_{p=1}^{m} a_{p}$. Let $G$ be the disjoint union of $a_{q}$ copies of $S_{1, q}$ for $q \in\{0, \ldots, m\}$. Then $a_{1, q}(G)=a_{q}$ for $q \in\{0, \ldots, m\}$ and $a_{p, q}(G)=0$ for all $p \neq 1, q$ or $p=1, q>m$. Similarly, let $G^{\prime}$ be the disjoint union of $a_{q}^{\prime}$ copies of $S_{1, q}$ for $q \in\{1, \ldots, m\}$. Then $a_{1, q}\left(G^{\prime}\right)=a_{q}^{\prime}$ for $q \in\{1, \ldots, m\}$ and $a_{p, q}\left(G^{\prime}\right)=0$ for all $p \neq 1, q$ or $p=1, q=0$ or $p=1, q>m$. Then for all $i \in \mathbb{N}$ we have

$$
\operatorname{hom}\left(S_{i, 0}, G\right)=\sum_{q=0}^{m} a_{q}=\sum_{q=1}^{m} a_{q}^{\prime}=\operatorname{hom}\left(S_{i, 0}, G^{\prime}\right)
$$

and, for $j \in[m]$,

$$
\operatorname{hom}\left(S_{i, j}, G\right)=\sum_{q=1}^{m} q^{j} a_{q}=\sum_{q=1}^{m} q^{j} a_{q}^{\prime}=\operatorname{hom}\left(S_{i, j}, G^{\prime}\right) .
$$

Thus $\operatorname{hom}(F, G)=\operatorname{hom}\left(F, G^{\prime}\right)$ for all $F \in \mathcal{F}$.

However, $G$ contains a copy of $S_{1,0}$, whereas $G^{\prime}$ does not. Thus $G$ satisfies the $\mathrm{FO}_{2-}$ sentence

$$
\exists x(\gamma(x)=\circ \wedge \exists y(E(x, y) \wedge \gamma(y)=\circ) \wedge \neg \exists y(E(x, y) \wedge \gamma(y)=\bullet))
$$

and $G^{\prime}$ does not. Hence $G$ and $G^{\prime}$ are not $\mathrm{FO}^{2}$-equivalent.

\subsection{Relational Structures}

Our main result extends from graphs to arbitrary relational structures. The definition of elimination forests and hence of tree depth can be extended in a straightforward way. Lemma 2.3, the inductive characterisation of tree depth, does not generalise directly, but can be adapted: when deleting the root, rather than removing all tuples that contain the root from all relations, we need to add relations of smaller arity and keep the remaining tuples after deleting the root. A similar adaptation will be necessary in the definition of $G \imath v$ in Section 4.1. It needs to be checked that Lemma 3.1 still holds with the new definitions - it does - , the rest of the proof goes through nearly unchanged.

It would be interesting to work out an extension of the main theorem to weighted graphs, yielding homomorphisms whose weight is the product of the edges weights in its image. Such an extension would require a suitable extension of the logic. We leave this for future work.

\subsection{Complexity}

Böker, Chen, Grohe, and Rattan [8] studied the computational complexity of homomorphism indistinguishability over classes $\mathcal{F}$ of graphs. Depending on $\mathcal{F}$, they found complexities ranging from polynomial time to undecidable. Notably, homomorphism 
indistinguishability over the class of all graphs is equivalent to isomorphism and hence decidable in quasi-polynomial time [4].

It is a consequence of our main theorem that for every $k$, homomorphism indistinguishability over $\mathcal{T} \mathcal{D}_{k}$ is decidable in polynomial time, or more precisely, time $n^{O(k)}$, because $C_{k}$-equivalence is decidable in this time. Probably the easiest way to see this is via the bijective pebble game: given graphs $G, G^{\prime}$, by induction on $\ell$ we can compute the partition of $V(G)^{k-\ell} \cup V\left(G^{\prime}\right)^{k-\ell}$ such that Duplicator wins the $\ell$-move bijective pebble game with initial position $\boldsymbol{x}, \boldsymbol{x}^{\prime}$ if and only $\boldsymbol{x}, \boldsymbol{x}^{\prime}$ belong to the same class of the partition.

We leave open the question whether homomorphism indistinguishability over $\mathcal{T} \mathcal{D}_{k}$ is fixed-parameter tractable when parameterised by $k$. We conjecture that it is not.

\section{Concluding Remarks}

We characterise equivalence in the counting extension of first-order logic, parameterised by quantifier rank, in terms of homomorphism indistinguishability over graphs of bounded tree depth. While a result along these lines may not be unexpected, it is surprising that we obtain such a tight and clean correspondence between quantifier rank and tree depth.

An interesting aspect of the correspondence between homomorphism counts and logical equivalence is that homomorphism counts also give us a natural notion of distance and similarity between graphs via distances between the homomorphism vectors $\operatorname{HOM}_{\mathcal{F}}(G)$ in suitable inner-product spaces. Through the translation between logic and homomorphism counts, such distance measures between graphs give us notions of "approximate logical equivalence" and possibly "approximate logical satisfiability", which in times of uncertain data seems very desirable and deserves further exploration.

\section{References}

[1] A. Atserias, Laura Mančinska, D.E. Roberson, R. Šámal, S. Severini, and A. Varvitsiotis. Quantum and non-signalling graph isomorphisms. Journal of Combinatorial Theory, Series B, 136:289-328, 2019.

[2] A. Atserias and E. Maneva. Sherali-Adams relaxations and indistinguishability in counting logics. SIAM Journal on Computing, 42(1):112-137, 2013.

[3] A. Atserias and J. Ochremiak. Definable ellipsoid method, sums-of-squares proofs, and the isomorphism problem. In Proceedings of the 33rd Annual ACM/IEEE Symposium on Logic in Computer Science, pages 66-75, 2018.

[4] L. Babai. Graph isomorphism in quasipolynomial time. In Proceedings of the 48th Annual ACM Symposium on Theory of Computing (STOC '16), pages 684-697, 2016 .

[5] M. Bannach and T. Tantau. Parallel multivariate meta-theorems. In J. Guo and D. Hermelin, editors, Proceedings of the 11th International Symposium on Parameterized and Exact Computation, volume 63 of LIPIcs, pages 4:1-4:17. Schloss Dagstuhl - Leibniz-Zentrum für Informatik, 2016. 
[6] C. Berkholz and M. Grohe. Limitations of algebraic approaches to graph isomorphism testing. In M.M. Halldórsson, K. Iwama, N. Kobayashi, and B. Speckmann, editors, Proceedings of the 42nd International Colloquium on Automata, Languages and Programming, Part I, volume 9134 of Lecture Notes in Computer Science, pages 155-166. Springer Verlag, 2015.

[7] J. Böker. Structural similarity and homomorphism counts. Master Thesis at RWTH Aachen, 2018.

[8] J. Böker, Y. Chen, M. Grohe, and G. Rattan. The complexity of homomorphism indistinguishability. In P. Rossmanith, P. Heggernes, and J.-P. Katoen, editors, Proceedings of the 44th International Symposium on Mathematical Foundations of Computer Science, volume 138 of Leibniz International Proceedings in Informatics (LIPIcs), pages 54:1-54:13. Schloss Dagstuhl-Leibniz-Zentrum fuer Informatik, 2019.

[9] C. Borgs, J. Chayes, L. Lovász, V. Sós, B. Szegedy, and K. Vesztergombi. Graph limits and parameter testing. In Proceedings of the 38th Annual ACM Symposium on Theory of Computing, pages 261-270, 2006.

[10] J. Bulian and A. Dawar. Graph isomorphism parameterized by elimination distance to bounded degree. In M. Cygan and P. Heggernes, editors, Proceedings of the 9th International Symposium on Parameterized and Exact Computation, volume 8894 of Lecture Notes in Computer Science, pages 135-146. Springer Verlag, 2014.

[11] J. Cai, M. Fürer, and N. Immerman. An optimal lower bound on the number of variables for graph identification. Combinatorica, 12:389-410, 1992.

[12] Y. Chen and J. Flum. Tree-depth, quantifier elimination, and quantifier rank. In Proceedings of the 33rd Annual ACM/IEEE Symposium on Logic in Computer Science, pages 225-234, 2018.

[13] H. Dell, M. Grohe, and G. Rattan. Lovász meets Weisfeiler and Leman. In I. Chatzigiannakis, C. Kaklamanis, D. Marx, and D. Sannella, editors, Proceedings of the 45th International Colloquium on Automata, Languages and Programming (Track A), volume 107 of LIPIcs, pages 40:1-40:14. Schloss Dagstuhl - Leibniz-Zentrum für Informatik, 2018.

[14] Z. Dvorák. On recognizing graphs by numbers of homomorphisms. Journal of Graph Theory, 64(4):330-342, 2010.

[15] H.-D. Ebbinghaus, J. Flum, and W. Thomas. Mathematical Logic. Springer Verlag, 2nd edition, 1994.

[16] M. Elberfeld, M. Grohe, and T. Tantau. Where first-order and monadic secondorder logic coincide. ACM Transaction on Computational Logic, 17(4), 2016. Article No. 25 . 
[17] M. Elberfeld, A. Jakoby, and T. Tantau. Algorithmic meta theorems for circuit classes of constant and logarithmic depth. In C. Dürr and T. Wilke, editors, Proceedings of the 29th International Symposium on Theoretical Aspects of Computer Science, volume 14 of LIPIcs, pages 66-77. Schloss Dagstuhl - Leibniz-Zentrum fuer Informatik, 2012.

[18] J. Flum and M. Grohe. The parameterized complexity of counting problems. SIAM Journal on Computing, 33(4):892-922, 2004.

[19] E. Grädel, M. Grohe, B. Pago, and W. Pakusa. A finite-model-theoretic view on propositional proof complexity. Logical Methods in Computer Science, 15(1):4:14:53, 2019.

[20] M. Grohe and M. Otto. Pebble games and linear equations. Journal of Symbolic Logic, 80(3):797-844, 2015.

[21] L. Hella. Logical hierarchies in PTIME. Information and Computation, 129:1-19, 1996.

[22] N. Immerman. Expressibility as a complexity measure: results and directions. In Proceedings of the 2nd IEEE Symposium on Structure in Complexity Theory, pages 194-202, 1987.

[23] N. Immerman and E. Lander. Describing graphs: A first-order approach to graph canonization. In A. Selman, editor, Complexity theory retrospective, pages 59-81. Springer-Verlag, 1990.

[24] N.M. Kriege, F.D Johansson, and C. Morris. A survey on graph kernels. ArXiv, arXiv:1903.11835 [cs.LG], 2019.

[25] D. Kuske and N. Schweikardt. First-order logic with counting. In Proceedings of the 32nd ACM-IEEE Symposium on Logic in Computer Science, 2017.

[26] D. Kuske and N. Schweikardt. Gaifman normal forms for counting extensions of first-order logic. In I. Chatzigiannakis, C. Kaklamanis, D. Marx, and D. Sannella, editors, Proceedings of the 45th International Colloquium on Automata, Languages, and Programming, volume 107 of LIPIcs, pages 133:1-133:14. Schloss Dagstuhl Leibniz-Zentrum für Informatik, 2018.

[27] L. Libkin. On counting and local properties. In Proceedings of the 13th IEEE Symposium on Logic in Computer Science, pages 501-512, 1998.

[28] L. Libkin. Logics with counting, auxiliary relations, and lower bounds for invariant queries. In Proceedings of the 14th IEEE Symposium on Logic in Computer Science, 1999. 
[29] L. Libkin. Logics capturing local properties. In Horst Reichel and Sophie Tison, editors, 17th Annual Symposium on Theoretical Aspects of Computer Science, volume 1770 of Lecture Notes in Computer Science, pages 217-229. Springer-Verlag, 2000 .

[30] L. Lovász. Operations with structures. Acta Mathematica Hungarica, 18:321-328, 1967.

[31] L. Lovász. Large Networks and Graph Limits. American Mathematical Society, 2012.

[32] L. Lovász and B. Szegedy. Limits of dense graph sequences. Journal of Combinatorial Theory, Series B, 96(6):933-957, 2006.

[33] T. Maehara and H. NT. A simple proof of the universality of invariant/equivariant graph neural networks. ArXiv (CoRR), arXiv:1910.03802v1 [cs.LG], 2019.

[34] P. Malkin. Sherali-adams relaxations of graph isomorphism polytopes. Discrete Optimization, 12:73-97, 2014.

[35] L. Mančinska and D.E. Roberson. Quantum isomorphism is equivalent to equality of homomorphism counts from planar graphs. ArXiv, arXiv:1910.06958v2 [quant-ph], 2019 .

[36] C. Morris, M. Ritzert, M. Fey, W. Hamilton, J.E. Lenssen, G. Rattan, and M. Grohe. Weisfeiler and leman go neural: Higher-order graph neural networks. In Proceedings of the 33rd AAAI Conference on Artificial Intelligence, volume 46024609. AAAI Press, 2019.

[37] J. Nešetřil and P. Ossona de Mendez. Linear time low tree-width partitions and algorithmic consequences. In Proceedings of the 38th ACM Symposium on Theory of Computing, pages 391-400, 2006.

[38] J. Nešetřil and P. Ossona de Mendez. Sparsity. Springer-Verlag, 2012.

[39] N. Schweikardt. Local normal forms and their use in algorithmic meta theorems (invited talk). In Proceedings of the 34th Annual ACM/IEEE Symposium on Logic in Computer Science, pages 1-3, 2019.

[40] N. Shervashidze, P. Schweitzer, E.J. van Leeuwen, K. Mehlhorn, and K.M. Borgwardt. Weisfeiler-Lehman graph kernels. Journal of Machine Learning Research, 12:2539-2561, 2011.

[41] S. Toda. PP is as hard as the polynomial-time hierarchy. SIAM Journal on Computing, 20(5):865-877, 1991. 